Pacific Journal of Mathematics

THE SECOND CONJUGATE SPACE OF A BANACH ALGEBRA 


\title{
THE SECOND CONJUGATE SPACE OF A BANACH ALGEBRA AS AN ALGEBRA
}

\author{
Paul Civin and Bertram Yood
}

1. Introducion. A procedure has been given by Arens $[1,2]$ for defining a multiplication in the second conjugate space of a Banach algebra which makes that space into another Banach algebra. This idea was used with great effectiveness by Day [3] in his study of amenable semigroups.

We undertake here a rather systematic study of this notion. We begin in $\S 3$ with a discussion of the second conjugate space $L^{* *}(\mathbb{( S )})$ of the group algebra $L(\mathbb{B})$ of a locally compact group $\left(\mathbb{S}\right.$ and its radical $\mathfrak{R}^{* *}$. Suppose that $\mathbb{B S}$ is abelian and infinite. It is shown that $L^{* *}(\mathbb{S})$ is never semi-simple and never commutative; if $(B)$ is compact then $\mathfrak{R}^{* *}$ is the annihilator in $L^{* *}(\mathbb{S})$ of that subset of the first conjugate space $L^{*}(\mathbb{B})$ which can be identified with the continuous functions on (S). For any locally compact abelian group $\left(\mathfrak{S}\right.$ let $\mathfrak{Y}$ be the subspace of $L^{*}(\mathbb{S})$ that may be identified with the almost periodic functions on $\mathbb{B}$, and let $\mathbb{S}$ be the subspace of $L^{*}(\mathbb{S})$ that may be identified with the continuous functions on $(\$)$ vanishing at infinity. Let $\mathfrak{Y}^{\perp}$ and $\mathbb{F}^{\perp}$ denote respectively the annihilators of $\mathfrak{Y}$ and $\mathfrak{C}$ in $L^{* *}(\mathbb{S})$. Then $L^{* *}(\mathfrak{S}) / \mathfrak{Y}^{\perp}$ is isometrically isomorphic as a Banach algebra to the measure algebra on the almost periodic compactification of $\mathbb{B}$, and $L^{* *}(\mathbb{S}) / \mathbb{S}^{\perp}$ is isometrically isomorphic to the measure algebra on $(S)$. It is then abundently clear that the Arens multiplication in $L^{* *}(\mathbb{S})$ is intimately connected with much studied objects defined in terms of $\mathbb{S}$.

In $\S 4$ we observe a phenomenon which does not hold in the group algebra case. In the latter case we started with a commutative, semisimple Banach algebra $B=L(\$)$ and obtained a second conjugate space $B^{* *}$ neither commutative nor semi-simple. Here we give of an example where $B$ is commutative and semi-simple and $B^{* *}$ is not semi-simple but commutative.

We can consider $B$ as embedded in $B^{* *}$ in the canonical way. In $\S 5$ it is shown, for example, that each regular maximal (left, right or two-sided) and each primitive ideal is contained in an ideal of the same type in $B^{* *}$. Also if $B$ is commutative its radical is contained in the radical of $B^{* *}$.

In $\S 6$ it is shown that if $T$ is a continuous homomorphism of $B_{1}$ into $B_{2}$, where $B_{k}$ is a Banach algebra, then $T^{* *}$ is continuous homomorphism of $B_{1}^{* *}$ into $B_{2}^{* *}$ where these are considered as algebras.

Received August 10,1960. This research was supported by the National Science Foundation, Grant NSF-G-5865. 
Curiously the analogous result fails to be true for anti-homomorphisms. In $\S 7$ it is observed that, as a consequence of the work of Sherman and Takeda [12], $B^{* *}$ is a $B^{*}$-algebra in the Arens multiplication for every $B^{*}$-algebra $B$.

2. Notation. Let $B$ be a Banach algebra (over the real or complex field). Let $B^{*}$ and $B^{* *}$ denote the first and second conjugate spaces of $B$. Let $x, y, \cdots$ denote elements of $B ; f, g, \cdots$ denote elements of $B^{*} ; F, G, \cdots$ denote elements of $B^{* *}$. For each $f \in B^{*}, x \in B$ we define $\langle f, x\rangle \in B^{*}$ be the rule $\langle f, x\rangle(y)=f(x y), y \in B$. For each $F \in B^{* *}$, $f \in B^{*}$ we define $[F, f] \in B^{*}$ by the rule $[F, f](x)=F(\langle f, x\rangle), x \in B$. For each pair of $F, G \in B^{* *}$ we define $F G \in B^{* *}$ by the rule $F G(f)=$ $F([G, f])$. These notions were introduced by Arens $[1,2]$ who showed the definition of $F G$ as the product of $F$ and $G$ yields an associative multiplication on $B^{* *}$ which makes $B^{* *}$ into a Banach algebra. Throughout we let $\pi$ denote the natural embedding of $B$ into $B^{* *}$. As noted by Arens [1], $\pi$ is an isometric isomorphism when $B^{* *}$ is considered as a Banach algebra under the above definition of multiplication.

For some purposes Arens [2] considered also the following definition of multiplication in $B^{* *}$. For each $f \in B^{*}, x \in B$ define $\langle f \mid x\rangle \in B^{*}$ by the rule $\langle f \mid x\rangle(y)=f(y x), y \in B$. For each $F \in B^{* *}, f \in B^{*}$ define $[F \mid f] \in B^{*}$ by the rule $[F \mid f](x)=F(\langle f \mid x\rangle), x \in B$. Finally for $F \in B^{* *}, G \in B^{* *}$ define $F \cdot G \in B^{* *}$ by the rule $F \cdot G(f)=F([G \mid f]), f \in B^{*}$. Again the definition of $F \cdot G$ as a product makes $B^{* *}$ into a Banach algebra. Arens calls the multiplication in $B$ regular provided $F \cdot G=G F$ for all $F, G \in B^{* *}$. Clearly, if $B$ is commutative, $B^{* *}$ is commutative under either definition of multiplication if and only if the multiplication in $B$ is regular.

As was noted in [2] the multiplication $F G$ is $w^{*}$-continuous in $F$ for fixed $G \in B^{* *}$ and $\pi(x) G$ is $w^{*}$-continnous in $G$ for fixed $x \in B$. If the multiplication in $B$ is regular, then $F G$ is also $w^{*}$-continuous in $G$ for fixed $F$.

We use $\Re^{* *}$ to denote the radical of the second conjugate space of the algebra under consideration, regardless of the symbol use to denote that algebra. We also use the symbol $\mathfrak{Y}$ for the closed subspace of the first conjugate space of a Banach algebra generated by the multiplicative linear functionals on the algebra, regardless of the symbol used for the original algebra.

3. Group algebras. The principal object of concern in this section is the second conjugate space, $L^{* *}(\mathbb{S})$, of the group algebra, $L(\mathbb{S})$, of a locally compact group \&S (with respect to right Haar measure). We consider $L^{* *}(\$)$ as a Banach algebra under the definitions of $\S 2$.

We consider first the case of a discrete infinite group $(B)$. In this connection see Day [3]. As in [3] we define the operator $l_{\sigma}, \sigma \in \mathbb{B}$, on 
$L^{*}(\mathbb{S})$ by the rule $l_{\sigma}(f)(\gamma)=f(\sigma \gamma), f \in L^{*}(\mathbb{S}), \gamma \in \mathbb{B}$. For $\gamma \in \mathbb{B}$ let $x^{\gamma}$ be the characteristic function of the singleton $\gamma$ considered as an element of $L(\$)$ and let $f_{\gamma}$ be that characteristic function considered as an element of $L^{*}(\mathfrak{S})$. Since $L^{*}(\mathfrak{S})$ may be identified with the bounded complexvalued functions on $\mathbb{B}$ and, for $g \in L^{*}(\mathbb{S})$, the value of $g\left(x^{\gamma}\right)$ is the same as $g(\gamma), \gamma \in \mathbb{B}$, after the identification, we shall use the symbols $g\left(x^{\gamma}\right)$ and $g(\gamma)$ interchangeably. Let $\mathfrak{\Im}=\left\{F \in L^{* *}(\mathfrak{S}) \mid F\left(l_{\gamma} f\right)=F(f)\right.$, for all $\left.\gamma \in \mathbb{S}, f \in L^{*}(\mathbb{S})\right\}$. Let $e$ be the element in $L^{*}(\mathbb{S})$ corresponding to the function identically one on $\mathbb{S}$. Let $\Im_{1}=\{F \in \Im \mid F(e)=0\}$. The following formulas were established in [3, pp. 527, 530].

$$
\begin{aligned}
{[F, f](\gamma) } & =F\left(l_{\gamma} f\right), \quad \gamma \in \mathbb{S}, \quad F \in L^{* *(\mathbb{S})} . \\
G F & =G(e) F, F \in \mathfrak{F}, G \in L^{* *}(\mathbb{S}) .
\end{aligned}
$$

The second conjugate space $L^{* *(\$ 3)}$ can be identified with the space of all bounded complex-valued finitely additive set functions defined for all subsets of (S).

It is clear from formula (3.2) that $\mathfrak{\Im}$ and $\mathfrak{\Im}_{1}$ are left ideals of $L^{* *}(\$)$. Let $F \in \mathfrak{F}$ and $G \in L^{* *}(\mathbb{S})$. For $\alpha, \beta \in \mathbb{S},\left[G, l_{\alpha} f\right](\beta)=G\left(l_{\beta} l_{\alpha}(f)\right)=G\left(l_{\alpha \beta} f\right)$ whereas $l_{\alpha}[G, f](\beta)=[G, f](\alpha \beta)=G\left(l_{\alpha \beta} f\right)$. Therefore $F G\left(l_{\alpha} f\right)=F\left(\left[G, l_{\alpha} f\right]\right)=$ $F\left(l_{\alpha}[G, f]\right)=F G(f)$. Thus $\Im$ is a two-sided ideal of $L^{* *}(\mathbb{S})$. Next let

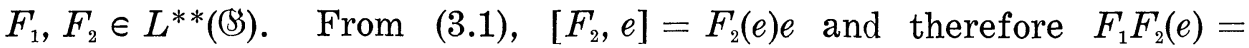
$F_{1}(e) F_{2}(e)$. It follows that $\mathfrak{\Im}_{1}$ is also a two-sided ideal of $L^{* *}(\mathbb{S})$.

As in [3, p. 510] let $m(\$)$ be the space of all real-valued bounded functions on $(S)$ and $m(B) *$ be its conjugate space. Each $\mu \in m((S))^{*}$ gives rise to a functional $F \in L^{* *}(\mathbb{S})$ by the following rule. If $f \in L^{*}(\mathbb{S})$ write $f=f_{1}+i f_{2}$ where $f_{1}, f_{2} \in m(\mathbb{S})$ and set $F(f)=\mu\left(f_{1}\right)+i \mu\left(f_{2}\right)$. An invariant mean $\mu[3$, p. 514] gives rise to an $F \in \mathfrak{F}$ and two different invariant means $\mu_{1}$ and $\mu_{2}$ correspond to different functionals $F_{1}$ and $F_{2}$ by this process.

3.1. THeOREM. Let (S) be an infinite discrete group, and let $L(\$ \$)$ be its group algebra. Under any of the following conditions, $L^{* *}(\mathbb{S})$ is not semi-simple and is not commutative.

(i) The commutator chain, $\mathbb{S S} \supset \mathbb{S}_{1} \cdots$, ends at the identity subgroup in a finite number of steps; in particular when $\mathbb{B}$ is abelian.

(ii) (S) is an amenable group and contains an element of infinite order.

(iii) (S) is locally finite; that is, every finite subset of (B) generates a finite subgroup of $\mathbb{S}$.

Proof. For the notion of an amenable group see [3, p. 515]. According to see Day [3, p. 535] under any of the conditions (i), (ii), (iii) there are at least two distinct invariant means on $m(\mathfrak{S S})$. As noted above 
this shows there exists $F_{1}, F_{2} \in \Im$ where $F_{1}(e)=F_{2}(e)=1$ and $F_{1} \neq F_{2}$. Then $F_{1}-F_{2}$ is a nonzero element of $\Im_{1}$. Formula (3.2) shows that $\Im_{1}^{2}=(0)$. Hence $L^{* *}(\mathbb{S})$ is not semi-simple.

Also $\Im$ has only zero in common with the center of $L^{* *}(\mathbb{S})$. For let $0 \neq F \in \mathfrak{\Im}$. By the above there exists $G \in \mathfrak{\Im}$ such that $G(e)=1$ and $G, F$ are linearly independent. By (3.2), $F G=F(e) G$ and $G F=G(e) F$. Thus $F G \neq G F$.

In the special case of $(B)$, the additive group of integers where we make a detailed investigation (Theorem 3.5) it is shown that $\mathfrak{\Im}_{1}$ is a very small part of the radical of $L^{* *}(\mathbb{S})$.

3.2. THEOREM. Let (\$) be a discrete group, and let $L(\mathbb{B})$ be its group algebra. Let $\Re=\left\{F \in L^{* *}(\mathbb{S}) \mid\right.$ the set function $\mu$ corresponding to $F$ vanishes on finite sets in (B\}). Then $\Omega$ is a two-sided ideal of

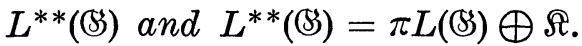

Proof. For $\gamma \in \mathbb{B}, l_{\gamma} f_{\sigma}(\beta)=f_{\sigma}(\gamma \beta), \beta \in \mathbb{B}$ and therefore $l_{\gamma} f_{\sigma}=f_{\gamma^{-1} \sigma} \cdot$ Now by formula $(3.1)\left[F, f_{\sigma}\right](\gamma)=F\left(f_{\gamma^{-1} \sigma}\right)$. Thus if $F \in \mathfrak{R},\left[F, f_{\sigma}\right]=0$ for all $\sigma \in \mathbb{S}$. Therefore $G F\left(f_{\sigma}\right)=0$ and $G F \in \Omega$ for all $G \in L^{* *}(\mathbb{S})$. Hence $\Re$ is a left ideal of $L^{* *}(\mathbb{\$})$.

Let $G \in L^{* *}(\$)$, and $F \in \Re$. For each $\gamma \in(\$)$, let $\varepsilon_{\gamma}$ be a complex number such that $\varepsilon_{\gamma} G\left(f_{\gamma}\right)=\left|G\left(f_{\gamma}\right)\right|$. Then for any finite subset $\gamma(1), \cdots, \gamma(n)$ of distinct elements of $B$,

$$
\sum_{k=1}^{n} G\left(\varepsilon_{\gamma(k)} f_{\gamma(k)}\right)=\sum_{k=1}^{n}\left|G\left(f_{\gamma(k)}\right)\right| \leqq\|G\|\left\|\sum_{k=1}^{n} \varepsilon_{\gamma(k)} f_{\gamma(k)}\right\| \leqq\|G\| .
$$

This shows that $G\left(f_{\gamma}\right) \neq 0$ for at most a countably infinite set $\gamma(1), \gamma(2), \cdots$ of distinct elements of (\$), and for these $\sum_{k=1}^{\infty}\left|G\left(f_{\gamma(k)}\right)\right|<\infty$. Fix $\sigma \in(B)$. For $k=1,2, \cdots$ define $g_{k}$ on \&s by the rule $g_{k}(\alpha)=G\left(f_{\alpha^{-1} 1_{\sigma}}\right)$ for $\alpha=$ $\sigma(\gamma(j))^{-1}, j=1, \cdots, k$, and $g_{k}(\alpha)=0$ otherwise. Clearly $g_{k} \in L^{*}(\mathcal{G})$. Since $\left[G, f_{\sigma}\right](\alpha)=G\left(f_{\alpha^{-1} \sigma}\right)$ we have $\left\|g_{k}-\left[G, f_{\sigma}\right]\right\|=\sup _{j>k}\left|G\left(f_{\gamma(j)}\right)\right| \rightarrow 0$. Then as $F\left(g_{k}\right)=0$, we have $F\left[G, f_{\sigma}\right]=0$ or $F G\left(f_{\sigma}\right)=0$. Since $\sigma$ was arbitrary in $(S)$, we see that $\Re$ is a right ideal.

Let $G \in L^{* *}(\mathbb{S})$. As was noted above, $G\left(f_{\sigma}\right) \neq 0$, for at most a countable set $\gamma(j), j=1,2, \cdots$, of distinct elements of $\mathbb{S B}$ and $\sum_{j=1}^{\infty}\left|G\left(f_{\gamma(j)}\right)\right|<\infty$. Consequently if we define $x$ by the rule $x(\gamma)=G\left(f_{\gamma}\right)$, then $x \in L(\mathbb{S})$. Also $(G-\pi(x))\left(f_{\gamma}\right)=0$ or $G-\pi x \in \Re$. Since $\Re \cap \pi L((\$))=(0)$, we see that $L^{* *}(\mathbb{S})=\pi L(\mathbb{S}) \oplus \Re$.

3.3. THEOREM. Let \&S be an infinite discrete group and $\Re$ be the ideal of Theorem 3.2. Then $\Re^{* *} \subset \Re$. Let $\mathbb{B}$ be an amenable group. Then $\Re$ properly contains $\mathfrak{R}^{* *}$ and $L^{* *}\left(\mathbb{( S )} / \Re^{* *}\right.$ can be written as the direct sum of two ideals $\mathfrak{K}_{1} \oplus \mathfrak{F}_{2}$ uhere $\mathfrak{K}_{1}$ is a maximal two-sided ideal and $\mathfrak{S}_{2}$ is the set of all scalar multiples of a central idempotent. 
Proof. By Theorem 3.2, $\Omega$ is a two-sided ideal of $L^{* *}(\$)$ and $L^{* *}(\mathbb{S}) / \Omega$ is semi-simple. Thus $\Re$ contains the radical of $L^{* *}(\mathbb{S})$.

Suppose that $(3)$ is amenable so that $(B)$ has an invariant mean. This gives rise to $F_{0} \in \mathfrak{\Im}$ where $F_{0}(e)=1$. If, for $\sigma \in \mathbb{S}, F_{0}\left(f_{\sigma}\right)=a \neq 0$ then $F_{0}(g)=n a$ for the characteristic function $g$ of a set of $n$ elements and $F_{0}$ is not bounded. Therefore $F_{0} \in \Re$. But $F_{0}^{2}=F_{0}$ by formula (3.2) so that $F_{0} \notin \Re^{* *}$.

Let $\lambda$ denote the natural homomorphism of $L^{* *}(\mathbb{S})$ onto $L^{* *}\left((\mathbb{S}) / \Re^{* *}\right.$. For any $F \in \Im, G \in L^{* *}(\mathbb{S})$, we have $F G-G F \in \Im$ and $(F G-G F)(e)=0$. Then $F G-G F \in \mathfrak{\Im}_{1} \subset \Re^{* *}$ by Theorem 3.1. Hence $\lambda(\Im)$ lies in the center of $L^{* *}(\mathfrak{S}) / \Re^{* *}$. Let $F \in \mathfrak{\Im}$ where $\lambda(F) \neq 0$. This requires that $F(e) \neq 0$ and that $\lambda\left(F(e) F_{0}-F\right)=0$. It follows that the ideal $\mathfrak{S}_{2}=\lambda(\Im)$ consists of all scalar multiples of the central idempotent $\lambda\left(F_{0}\right)$. Moreover $\mathfrak{S}_{2}$ must be a minimal left, right and two sided ideal of the semi-simple algebra $L^{* *}(\mathbb{S}) / \Re^{* *}$ so that we can write that algebra as the direct sum $\mathfrak{C}_{1} \oplus \mathfrak{S}_{2}$ where $\mathfrak{K}_{1}$ is the annihilator of $\mathfrak{K}_{2}$ and $\mathfrak{C}_{1}$ is a maximal left, right and two sided ideal.

3.4. THEOREM. Let $\$ 3$ be a discrete group. Let $\Gamma$ be the $w^{*}$-closure in $L^{* *}(\mathbb{S})$ of $\left\{\pi\left(x^{\gamma}\right) \mid \gamma \in \mathbb{S}\right\}$. Then $\Gamma$, in its $w^{*}$-topology is the StoneCech compactification of $\mathbb{B S}$ and is, under the multiplication of $L^{* *}(\mathbb{S})$, a semigroup containing as a dense subsemigroup a copy of (S). If (5) contains an element of infinite order then right multiplication in $\Gamma$ is not continuous.

Proof. We can view $L^{*}(\$)$, the space of all bounded functions on (S) as a commutative Banach algebra of continuous bounded functions on (S) under the usual conventions. Now, for $\gamma \in \mathbb{S}, \pi\left(x^{\gamma}\right) f=f\left(x^{\gamma}\right)=f(\gamma)$, $f \in L^{*}(\mathbb{S})$ so that the functional $\pi\left(x^{\gamma}\right)$ on $L^{*}(\mathbb{S})$ is evaluation at the point $\gamma$. Then $\pi\left(x^{\gamma}\right)$ is a multiplicative linear functional on $L^{*}(\mathbb{S})$ and, moreover, the $w^{*}$-closure $\Gamma$ of the set of all $\pi\left(x^{\gamma}\right)$ is [7, p. 55] the set of all nonzero multiplicative linear functionals on $L^{*}(\mathbb{S})$. These are the $F \in L^{* *}(\mathbb{S})$ for which the corresponding bounded finitely additive set function $\mu$ takes on the values 0 and 1 only (see Šmulian [11]). In this connection note that if $\mu$ corresponds to $\pi\left(x^{\gamma}\right)$ then $\mu(\xi)=1$ if and only if $\gamma \in \mathbb{F}$. The other $F \in \Gamma$ correspond to $\mu$ which vanish on finite sets, i.e. all other $F \in \Gamma$ lie in $\Re$. Also, [6, p. 167], $\Gamma$ is the Stone-Čech compactification of $(B)$.

Let $F_{k} \in L^{* *}(\mathfrak{S})$ correspond to the set function $\mu_{k}, k=1,2,3$ where $F_{1}, F_{2} \in \Gamma$ and $F_{3}=F_{1} F_{2}$. We show that $F_{3} \in \Gamma$. Let $\mathfrak{Q}$ be any subset of $\mathbb{B S}$ and let $\phi_{\mathfrak{Q}} \in L^{*}(\mathbb{S})$ be the characteristic function of the set $\cong \subset \mathbb{B}$. Since $l_{\gamma}\left(\phi_{\mathfrak{Q}}\right)=\phi_{\gamma^{-1} \mathfrak{Q}}$ we have, by formula $(3.1), \quad\left[F_{2}, \phi_{\mathfrak{Q}}\right](\gamma)=\mu_{2}\left(\gamma^{-1} \mathfrak{Q}\right)$. Let $\psi$ be the characteristic function of the set of $\gamma$ for which $\mu_{2}\left(\gamma^{-1} \mathfrak{D}\right)=1$. Then 


$$
\begin{aligned}
\mu_{3}(\Omega) & =F_{3}\left(\phi_{\mathfrak{Q}}\right)=\int_{\mathscr{S}} \psi(\gamma) d \mu_{1}(\gamma) \\
& =\mu_{1}\left(\left\{\gamma \mid \mu_{2}\left(\gamma^{-1} \mathfrak{\bigcap}\right)=1\right\}\right) .
\end{aligned}
$$

Thus the set function $\mu_{3}$ cannot taken on values other then 0 or 1 . Clearly $\mu_{3}(\mathbb{S})=1$ so that $F_{3} \in \Gamma$. Hence $\Gamma$ is a semigroup in the multiplication of $L^{* *}(\mathbb{S})$. Let $\beta, \gamma \in \mathbb{B}$. It is easy to see that $\pi\left(x^{\beta}\right) \pi\left(x^{\gamma}\right)=\pi\left(x^{\beta \gamma}\right)$. Thus a copy of $(B)$ is a dense subsemigroup of $\Gamma$.

Suppose (\$) contains an element of infinite order. Then (\$) contains a copy $\mathfrak{S}$ of the additive group of integers. Let $F \in \Gamma$ correspond to a set function $\mu$ vanishing for all sets in $(\mathbb{S}$ disjoint with $\mathfrak{S}$ and let $\gamma \in \mathfrak{S}$. We show first that $\pi\left(x^{\gamma}\right) F=F \pi\left(x^{\gamma}\right)$. For any $f \in L^{*}(\mathbb{S}), \pi\left(x^{\gamma}\right) F(f)=$ $\pi\left(x^{\gamma}\right)[F, f]=F\left(\left\langle f, x^{\gamma}\right\rangle\right)$ while $\left.\left.F \pi\left(x^{\gamma}\right)(f)=F(] \pi\left(x^{\gamma}\right), f\right]\right)$. It is then sufficient to show that, for each $\alpha \in \mathfrak{S},\left\langle f, x^{\gamma}\right\rangle(\alpha)=\left[\pi\left(x^{\gamma}\right), f\right](\alpha)$. The left hand side is $f(\gamma \alpha)$ while the right hand side is $f(\alpha \gamma)$. Since $\gamma$ and $\alpha$ permute, we have the desired result. Next we show that each such $F$ is in the $w^{*}$-closure of $\left\{\pi\left(x^{\gamma}\right) \mid \gamma \in \mathfrak{S}\right\}$. Let $\mu_{0}$ be the set function $\mu$ restricted to subsets of $\mathfrak{S}$. Since $\mu_{0}$ corresponds [11] to a multiplicative linear functional on the Banach algebra of all bounded complex-valued functions on $\mathfrak{S}$, there is a directed set $\gamma(j)$ of elements of $\mathfrak{S}$ such that

$$
\lim _{j} g(\gamma(j))=\int_{\mathfrak{g}} g(\gamma) d \mu_{0}(\gamma)
$$

for all bounded functions on $\mathfrak{S}$. Let $f$ be a bounded function on (S). From (3.4) we obtain

$$
\lim _{j} \pi\left(x^{\gamma(\mathcal{J})}\right)(f)=\int_{\mathfrak{S}} f(\gamma) d \mu_{0}(\gamma)=\int_{\mathscr{S}} f(\gamma) d \mu(\gamma)=F(f) .
$$

We define $F_{1} \in \Gamma$ where the corresponding set function $\mu_{1}$ is zero on finite subsets of $\mathfrak{Q}, \mu_{1}(\mathfrak{P})=1$ where $\mathfrak{P} \subset \mathfrak{S}$ is the set of positive integers. Note that $\mu_{1}$ being a $0-1$ set function is zero for sets disjoint with $\mathfrak{S}$. Define $F_{2} \in \Gamma$ to correspond to the set function $\mu_{2}$ where $\mu_{2}(\mathfrak{Q})=\mu_{1}\left(\mathfrak{Q}^{-1}\right)$ for any subset $\mathfrak{Q}$ of $\mathbb{S}$. If $\alpha \in \mathbb{S}, \alpha \notin \mathfrak{Z}$, the set $\alpha^{-1 \mathfrak{P}}$ lies outside $\mathfrak{S}$. If $\alpha \in \mathfrak{C}$ then $\alpha^{-1 \mathfrak{B}}=\mathfrak{P}$ modulo a finite set. Thus $\mu_{1}\left(\alpha^{-1 \mathfrak{P}}\right)=1$ if and only if $\alpha \in \mathfrak{S}$ and otherwise $\mu_{1}\left(\alpha^{-1 \mathfrak{P})}\right)=0$. According to formula (3.3),

$$
F_{2} F_{1}\left(\phi_{\mathfrak{S}}\right)=\mu_{2}(\mathfrak{S})=1 \text {. }
$$

Note that $\mu_{2}(\mathfrak{P})=0$. A calculation similar to (3.6) yields

$$
F_{1} F_{2}\left(\phi_{\mathfrak{\beta}}\right)=0 \text {. }
$$

By our earlier remarks there exists a direct set $\gamma(j)$ of elements of $\mathfrak{L}$ such that $\pi\left(x^{\gamma(j)}\right) \rightarrow F_{1}$ in the $w^{*}$-topology. Then $\pi\left(x^{\gamma(j)}\right) F_{2} \rightarrow F_{1} F_{2}$. As noted above, $\pi\left(x^{\gamma(j)}\right) F_{2}=F_{2} \pi\left(x^{\gamma(j)}\right)$. If multiplication on the right were 
continuous in $\Gamma$ we would have $F_{1} F_{2}=F_{2} F_{1}$. This contradicts (3.6) and (3.7).

The semigroup $\Gamma$ has the further property that $G_{1} G_{2}=\pi\left(x^{\beta}\right), \beta \in(\mathbb{S})$ for $G_{1}, G_{2} \in \Gamma$ if and only if each $G_{k}$ is of the form $\pi\left(x^{\alpha}\right), \alpha \in \mathbb{B}$. For suppose $G_{1} G_{2}=\pi\left(x^{\beta}\right)$ where $G_{k}$ corresponds to the $0-1$ set function $\nu_{k}$, $k=1$, 2. By (3.3), $\nu_{1}\left(\left\{\gamma \mid \nu_{2}\left(\gamma^{-1} \mathfrak{Q}\right)=1\right\}\right)=1$ if and only if $\beta \in \mathfrak{O}$. Applying this result to $\mathfrak{Q}$, the singleton $\{\beta\}$, we have a contradiction if $\nu_{2}$ vanishes for all singletons. Hence $G_{2}=\pi\left(x^{\alpha}\right)$ for some $\alpha \in(B)$. Then using the same $\supseteq$, we see that $1=\nu_{1}\left(\left\{\beta \alpha^{-1}\right\}\right)$ so that $G_{1}=\pi\left(x^{\delta}\right)$, where $\delta=\beta \alpha^{-1}$. In particular $\Gamma$ is never a group if $(\$)$ is infinite.

We shall see in Corollary 3.13 that the radical of $L^{* *}(\mathbb{S})$ is infinitedimensional for every locally compact abelian group which is not discrete. We conjecture this for all infinite locally compact abelian groups. However, in the infinite discrete case, we have been able to prove this only for (S) the additive group of integers.

3.5. THEOREM. Let (S) be the additive group of integers. Then the radical of $L^{* *}(\mathbb{S})$ is infinite-dimensional. In fact $\Re^{* *} / \Im_{1}$ is infinitedimensional.

Proof. Let $T$ be the operator on $(m)=L^{*}(\mathbb{S})$ defined by the rule $(T f)(n)=f(n+1)$. Let $e_{k}^{r} \in L^{*}(\$)$ for $k$ a positive integer and $r$ an integer be defined by the rule $e_{k}^{r}(n)=1$ if $n \equiv r \bmod k$ and $e_{k}^{r}(n)=0$ for

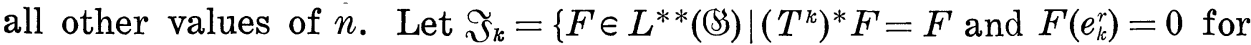
$r=0,1, \cdots, k-1\}$. For $k=1$ this is just the ideal $\mathfrak{\Im}_{1}$ defined earlier in the case of the present $(\$)$.

Let $F \in \widetilde{\Im}_{k}, f \in L^{*}(\mathbb{S})$ and $r, s$ be integers. First $\left\langle f, x^{r}\right\rangle(s)=f(r+s)$ so that $\left\langle f, x^{r}\right\rangle=T^{r} f$. Then $[F, f]\left(x^{r}\right)=\left(T^{r}\right)^{*} F(f)$. Let $\Sigma c_{r} x^{r}$ be an arbitrary element of $L(\mathbb{S})$. We have

$$
[F, f]\left(\Sigma c_{r} x^{r}\right)=\sum_{r} c_{r}\left(T^{r}\right)^{*} F(f) .
$$

Now since $F \in \Im_{k}$, this yields

$$
[F, f]=\sum_{r=0}^{k-1}\left(T^{r}\right)^{*} F(f) e_{k}^{r} .
$$

Let $G \in L^{* *((3)}$. From (3.8) we obtain

$$
G F=\sum_{r=0}^{k-1} G\left(e_{k}^{r}\right)\left(T^{r}\right)^{*} F .
$$

It follows readily from (3.9) that $\widetilde{\Im}_{k}$ is a left ideal of $L^{* *}(\mathbb{S})$ and that $\mathfrak{\Im}_{k}^{2}=(0)$. Hence each $\mathfrak{\Im}_{k}$ is contained in the radical of $L^{* *}(\mathbb{S})$.

Note that

$$
e_{2^{m}}^{r}=e_{2^{m+1}}^{r}+e_{2^{m+1}}^{r+2^{m}} \text {. }
$$


Let $F \in \Im_{2^{m}}$. Then $F\left(e_{2^{m}}^{r}\right)=0$ and, as $\left(T^{2^{m}}\right)^{*} F=F, F$ has the same value at each of the summands on the right of (3.10). It follows that $\mathfrak{\Im}_{2} m+1 \supset \mathfrak{\Im}_{2} m$. The desired result is established if we show each $\mathfrak{\Im}_{2}^{m+1}$ contains an element not in $\mathfrak{\Im}_{2} m$.

As noted in the proof of Theorem 3.1, there exist at least two distinct nonzero elements $F_{1}, F_{2} \in L^{* *}(\mathbb{S})$ where $F_{k}(e)=1, T^{*} F_{k}=F_{k}$, $k=1,2$. Let $U$ be the operator on $L^{*}(\mathbb{S})$ defined by the rule $U(f)(n)=$ $f\left(n 2^{m+1}\right)$. Let $H=U^{*}\left(F_{1}-F_{2}\right)$. In turn we have $\left(T^{2^{m+1}} f\right)(n)=f\left(n+2^{m+1}\right)$, $\left(U T^{2^{m+1}} f\right)(n)=f\left((n+1) 2^{m+1}\right)$ and thus $U T^{2^{m+1}}=T U$. Therefore $\left(T^{2^{m+1}}\right)^{*} H=$ $\left(T^{2^{m+1}}\right)^{*} U^{*}\left(F_{1}-F_{2}\right)=U^{*} T^{*}\left(F_{1}-F_{2}\right)=H$. Also $U\left(e_{2^{m+1}}^{r}\right)=0 \quad$ unless $r \equiv 0 \bmod 2^{m+1}$ in which case $U\left(e_{2 m+1}^{r}\right)=e$. Thus $H\left(e_{2^{m+1}}^{r}\right)=0$ for each $r$ and therefore $H \in \mathfrak{\Im}_{2 m+1}$. We shall show that $H \notin \mathfrak{\Im}_{2 m}$.

Select $f \in L^{*}(\mathbb{S})$ such that $F_{1}(f) \neq F_{2}(f)$. Define $g \in L^{*}(\mathbb{S})$ by the rule $g\left(2^{m+1} n\right)=f(n)$ and $g(s)=0$ otherwise. Clearly $U g=f$ so that $H(g) \neq 0$. Now $T^{2^{m}} g(k)=g\left(k+2^{m}\right)$. Therefore $U T^{2^{m}} g(k)=g\left(k 2^{m+1}+2^{m}\right)=0$. Hence $\left(T^{2^{m}}\right)^{*} H(g)=\left(F_{1}-F_{2}\right) U T^{2^{m}}(g)=0$. From this we see that $H \notin \Im_{2^{m}}$.

We next direct our attention to the case of $L^{* *}(\mathbb{S})$ where $\mathbb{B S}$ is a locally compact abelian group which is not discrete. Certain preliminary material will be needed for this purpose.

3.6. Lemma. Let $B$ be a Banach algebra, and let $f \in B^{*}$ be multiplicative on $B$. Then the functional $\phi \in B^{* * *}$ defined by $\phi(F)=F(f)$ is multiplicative on $B^{* *}$.

Proof. For $x, y \in B,\langle f, x\rangle(y)=f(x y)=f(x) f(y)$. Thus $\langle f, x\rangle=$ $f(x) f$. Next for $F \in B^{* *}$ and $x \in B,[F, f](x)=F(\langle f, x\rangle)=f(x) F(f)$, and consequently $[F, f]=F(f) f$. Therefore for any $F$ and $G$ in $B^{* *}$ $F G(f)=F([G, f])=F(f) G(f)$.

For a subspace $J$ of a Banach space $X$, we define $J^{\perp}=$ $\left\{z^{*} \in X^{*} \mid z^{*}(y)=0, y \in J\right\}$. For the definition of $\mathfrak{Y}$ see $\S 2$.

3.7. TheOREM. Let $B$ be a commutative Banach algebra. Then $\mathfrak{Y}^{\perp}$ is a two sided ideal of $B^{* *}$. Moreover, the algebra $B^{* *} / \mathfrak{Y}^{\perp}$ is commutative and semi-simple.

Proof. Let $F \in \mathfrak{Y}^{\perp}$ and let $G \in B^{* *}$. By Lemma 3.6, if $f$ is a multiplicative linear functional on $B$, then $F G(f)=F(f) G(f)=0$. The relation $F G(f)=0$ then holds for any linear combination of multiplicative linear functionals, and so, by continuity, for any $f \in \mathfrak{Y}$. Thus $F G \in \mathfrak{Y}^{\perp}$. The identical argument shows $G F \in \mathfrak{Y}^{\perp}$ and thus $\mathfrak{Y}^{\perp}$ is a two sided ideal of $B^{* *}$.

In view of the definition of multiplication in a quotient algebra, to show the commutativity of $B^{* *} / \mathfrak{Y}^{\perp}$, it is sufficient to show that for any pair $F, G \in B^{* *}, F G-G F \in \mathfrak{Y}^{\perp}$. For any multiplicative linear 
functional $f$, by Lemma 3.6, $F G(f)=F(f) G(f)=G F(f)$. Thus $F G-$ $G F \in \mathfrak{Y}^{\perp}$.

Let $\alpha$ denote the natural mapping of $B^{* *}$ onto $\left.B^{* *} / \mathfrak{Y}\right)^{\perp}$. For $f$ a multiplicative linear functional on $B$, define $f_{0}$ on $B^{* *} / \mathfrak{Y}^{\perp}$ by $f_{0}(F+\mathfrak{Y} \perp)=$ $F(f)$. The functional $f_{0}$ is clearly well defined. Also $f_{0}$ is multiplicative, since by Lemma $\left.3.6 f_{0}\left((F+\mathfrak{Y})^{\perp}\right)\left(G+\mathfrak{Y}^{\perp}\right)\right)=f_{0}\left(F G+\mathfrak{Y}^{\perp}\right)=(F G)(f)=$ $F(f) G(f)=f_{0}\left(F+\mathfrak{Y}^{\perp}\right) f_{0}\left(G+\mathfrak{Y}^{\perp}\right)$.

Suppose that $\alpha(F)$ is in the radical of $\left.B^{* *} / \mathfrak{Y}\right)^{\perp}$. Then for any multiplicative linear functional $f$ on $B, 0=f_{0}(\alpha(F))=F(f)$. Consequently $F \in \mathfrak{Y}^{\perp}$, so $\alpha(F)=0$. Thus $\left.B^{* *} / \mathfrak{Y}\right)^{\perp}$ is semi-simple.

We say that a Banach algebra $B$ has a weak right identity if there exists a net $\left\{e_{\alpha} \mid \alpha \in \mathfrak{A}\right\}$ in $B$ and an $M>0$ such that $\left\|e_{\alpha}\right\|<M, \alpha \in \mathfrak{A}$ and $\lim f\left(x e_{\alpha}-x\right)=0$ for each $x \in B, f \in B^{*}$.

3.8. Lemma. The Banach algebra $B$ has a weak right identity if and only if $B^{* *}$ has a right identity.

Proof. Suppose $B$ has a weak right identity $\left\{e_{\alpha} \mid \alpha \in \mathfrak{A}\right\}$, with $\left\|e_{\alpha}\right\|<M, \alpha \in \mathfrak{A}$. Since also $\left\|\pi e_{\alpha}\right\| \leqq M, \alpha \in \mathfrak{A}$, the $w^{*}$-compactness of the ball of radius $M$ in $B^{* *}$, implies the existence of a subnet $\left\{e_{\beta} \mid \beta \in \mathfrak{B}\right\}$ such that $w^{*}-\lim \pi e_{\beta}=E \in B^{* *}$. We shall show that $E$ is the required right identity for $B^{* *}$. Let $f \in B^{*}, x \in B$. Then $[E, f](x)=E(\langle f, x\rangle)=$ $\lim \pi e_{\beta}\langle f, x\rangle=\lim f\left(x e_{\beta}\right)=f(x)$. Consequtently $[E, f]=f$ for all $f \in B^{*}$. Thus $F E(f)=F([E, f])=F(f)$ and $F E=F$ for all $F \in B^{* *}$.

Suppose that $B^{* *}$ has a right identity $E$. By Goldstine's theorem [5, p. 424], there is a net $\left\{\pi e_{\alpha} \mid \alpha \in \mathfrak{2}\right\}$, with $\left\|\pi e_{\alpha}\right\| \leqq\|E\|, \alpha \in \mathfrak{A}$, and $w^{*}-\lim \pi e_{\alpha}=E$. Let $f \in B^{*}$. Since $E$ is a right identity in $B^{* *}$, $F(f)=F E(f)=F([E, f])$ for all $F \in B^{* *}$ and thus $[E, f]=f$. Hence for any $x \in B$, and $f \in B^{*}, f(x)=[E, f](x)=E(\langle f, x\rangle)=\lim \pi e_{\alpha}(\langle f, x\rangle)=$ $\lim f\left(x e_{\alpha}\right)$. Consequently $B$ has a weak right identity.

3.9. Lemma. If $B$ is a commutative Banach algebra then $\pi(B)$ is in the center of $B^{* *}$.

Proof. One verifies that, for $f \in B^{*}, x \in B$, we have $[\pi(x), f]=$ $\langle f, x\rangle$. Then $F \pi(x)(f)=F(\langle f, x\rangle)=[F, f](x)=\pi(x) F(f)$ for each $F \in B^{* *}$.

3.10. THEOREM. Let $B$ be a commutative semi-simple Banach algebra, and let $\mathfrak{F}$ be a closed linear manifold in $B^{*}$. The following statements are equivalent:

(i) For each $f \in B^{*}$, and $x \in B,\langle f, x\rangle \varepsilon$ ? .

(ii) $B^{* *} \Im^{\perp}=(0)$.

(iii) $F^{\perp} \pi(B)=(0)$. 
Proof. Suppose that (i) holds. Let $G \in B^{* *}$ and let $F \in \mathfrak{F}^{\perp}$. For any $f \in B^{*},[F, f]=0$, since $[F, f](x)=F(\langle f, x\rangle)=0$ for all $x \in B$. Thus $G F(f)=0$ for any $f \in B^{*}$ and so $G F=0$, and (i) implies (ii).

Suppose that (ii) holds. Let $F \in \mathfrak{F}^{\perp}$ and $x \in B$. Then $\pi(x) F=0$. However, by Lemma 3.9. $\pi(x)$ is in the center of $B^{* *}$ and thus $F \pi(x)=0$, and so (ii) implies (iii).

Suppose that (iii) holds. Let $f \in B^{*}$ and $x \in B$. For any $F \in \mathfrak{F}^{\perp}$, $0=F \pi(x)(f)=F([\pi(x), f])$, and thus $[\pi(x), f] \in \mathfrak{F}$. However, as noted in the proof of Lemma 3.9 , for $B$ commutative, $[\pi(x), f]=\langle f, x\rangle$, so $\langle f, x\rangle \varepsilon \mathfrak{F}$ and (iii) implies (i).

3.11. COROLlary. Under any of the conditions of Theorem 3.10, $\mathfrak{F}^{\perp}$ is contained in the radical of $B^{* *}$. In particular, if (1) is satisfied for $\mathfrak{F}=\mathfrak{Y}$, then $\mathfrak{F}^{\perp}$ is the radical of $B^{* *}$.

Proof. The first statement of the corollary is immediate from Theorem 3.10. The second statement is then a consequence of Theorem 3.7 , which implies that the radical of $B^{* *}$ is contained in $\mathfrak{F}^{\perp}$.

3.12. Theorem. Let (S) be a locally compact abelian group which is not discrete, and let $L(\mathbb{S})$ be the group algebra of $\left(\mathbb{S}\right.$. Then $L^{* *}(\mathbb{S})$ is not commutative and is not semi-simple.

Proof. Let $D$ be the closed subspace of $L^{*}(\mathbb{S})$ which can be identified with the collection of bounded continuous functions on (B). Since (8) is not discrete, it is an immediate consequence of the Hahn-Banach theorem that $\mathfrak{D}^{\perp} \neq(0)$.

We note for any $f \in L^{*}(\mathbb{S})$, and $x \in L(\mathbb{S})$, that $\langle f, x\rangle \varepsilon \mathbb{D}$. For if $y \in L($ (S) then

$$
\langle f, x\rangle(y)=f(x y)=\int f(\alpha) \int x\left(\alpha \beta^{-1}\right) y(\beta) d \beta d \alpha=\int\left\{\int f(\alpha) x\left(\alpha \beta^{-1}\right) d \alpha\right\} y(\beta) d \beta .
$$

Thus $\langle f, x\rangle$ may be identified with the function on (S) whose value at $\beta \in \mathbb{S}$ is $\int f(\alpha) x\left(\alpha \beta^{-1}\right) d \alpha$, and that function is continuous. We have thus shown that condition (i) of Theorem 3.10 is satisfied with $(\mathcal{D}$ playing the role of $\mathfrak{F}$. Thus by Corollary $3.11,(0) \neq \mathscr{D}^{\perp}$ is in the radical of $L^{* *}(\mathbb{S})$ and consequently $\left.L^{* *(\$)}\right)$ is not semi-simple.

To see that $L^{* *}(\mathbb{S})$ is not commutative let $F \neq 0, F \in \mathfrak{D}^{\perp}$. Since $L(\mathbb{S})$ has an approximate identity, Lemma 3.8 yields a right identity $E$ for $L^{* *(S)}$. Then $F=F E$, while from (ii) of Theorem $3.10, E F=0$.

3.13. CoRollaRy. Let (\$) be a locally compact abelian group which is not discrete, and let $L(\mathbb{S})$ be the group algebra of $\mathbb{B S}$. Then the 
radical of $L^{* *(\$)}$ is infinite-dimensional.

Proof. For any integer $n$, there are $n$ mutually disjoint compact neighborhoods in $\mathbb{B S}$, whose union is not all of (S). The characteristic functions of these sets thus correspond to $n$ elements of $L^{*}(\mathbb{S})$ which are linearly independent modulo $\mathfrak{D}$, in the notation of the proof of Theorem 3.12. Consequently, by the Hahn-Banach theorem, there are at least $n$ linearly independent elements in $\mathfrak{D}^{\perp}$, and thus in $\mathfrak{R}^{* *}$. Hence the radical of $L^{* *}(\mathbb{S})$ is infinite-dimensional.

3.14. THEOREM. Let (S) be an infinite locally compact abelian group, and let $L(\mathbb{S})$ be the group algebra of (\$). Then $L^{* *}(\mathbb{S})$ is not commutative and not semi-simple.

Proof. For (S) discrete, the result is a part of Theorem 3.1 and for (3) not discrete, the result is Theorem 3.12.

3.15. Theorem. Let (S) be a compact abelian group, and let $L(\mathbb{S})$ be the group algebra of (S). Then the radical of $L^{* *}(\mathbb{S})$ may be identified with any of the following sets:

(i) $\subseteq=\left\{F \in L^{* *}(\mathbb{S}) \mid L^{* *}(\mathbb{S}) F=(0)\right\}$

(ii) $\mathfrak{Y}^{\perp}$, where $\mathfrak{Y}$ is the closed subspace of $L^{*}(\mathbb{S})$ generated by the multiplicative linear functionals on $L(\mathbb{S})$.

(iii) $\mathfrak{D}^{\perp}$, where $\mathfrak{D}$ is the closed subspace of $L^{*}(\mathbb{S})$ which can be identified with the collection of continuous functions on $\mathbb{B}$.

Proof. If (5) where discrete, it would be finite since it is compact. Thus $L(\$)$ is finite-dimensional and is then isomorphic as an algebra to $L^{* *}(\mathbb{S})$, so the latter is semi-simple. In this case it is clear that each of the representations given for the radical of $L^{* *}(\mathbb{S})$ reduces to zero.

Suppose (S) is not discrete. From the proof of Theorem 3.12 and from the equivalences of Theorem 3.10, it follows that $\mathfrak{D}^{\perp} \subset \subseteq \subset \mathfrak{R}^{* *}$. Also from Theorem 3.7, we see that $L^{* *}((\mathfrak{S}) / \mathfrak{Y})^{\perp}$ is semi-simple so $\left.\Re^{* *} \subset \mathfrak{V}\right)^{\perp}$. However, the Peter-Weyl theorem [7, p. 155] asserts that $\mathscr{D}=\mathfrak{Y}$, and consequently $\mathfrak{D}^{\perp}=\mathfrak{Y}^{\perp}$. We thus see that $\mathfrak{D}^{\perp}=\mathfrak{S}=\mathfrak{R}^{* *}=\mathfrak{Y}^{\perp}$.

We shall show in Lemma 3.19 that the equality $\Re^{* *}=\mathfrak{Y}^{\perp}$ which was demonstrated for $L^{* *}(\mathbb{S})$ with (B) a compact abelian group fails when (B) is not compact.

3.16. Lemma. Let $B$ be a commutative semi-simple Banach algebra. Then there is a continuous isomorphism of $B$ into $B^{* *} / \mathfrak{Y}^{\perp}$.

Proof. Let $\alpha$ be the natural mapping of $B^{* *}$ onto $B^{* *} / \mathfrak{Y}{ }^{\perp}$. Since the composite mapping $\alpha \pi$ is clearly an algebra homomorphism, and is 
continuous, it sufficies to show that if $\alpha \pi(x)=0$, then $x=0$. Suppose $\alpha \pi(x)=0$. Then $\pi(x) \in \mathfrak{Y}$ and $0=\pi(x)(f)=f(x)$ for each multiplicative functional $f$. Thus, by the semi-simplicity of $B, x=0$.

The question whether the mapping $\alpha \pi$ is in general bicontinuous remains. The bicontinuity is clear if the spectral radius on $B$ is a complete norm. It is also possible to show the bicontinuity in concrete cases, where all the computations can be made. One such example is when $B$ is the collection of all complex-valued functions with $n$ continuous derivatives made into a Banach algebra in the usual way [6, p. 119]. We are grateful to the referee for the following example where $\alpha \pi$ is not bi-continuous. Let $C_{0}(R)$ be the algebra of all complex continuous functions on the reals $R$ vanishing at infinity with $\|f\|_{\infty}$ as the usual sup norm. Let $B$ be the subalgebra of $C_{0}(R)$ consisting of all absolutely integrable functions. We norm $B$ by setting $\|f\|=\|f\|_{\infty}+\|f\|_{1}$ where $\|f\|_{1}$ is the $L_{1}$ norm of $f$. In this norm $B$ is a Banach algebra. As a function algebra on $R, B$ is conjugate closed and adverse closed so that the multiplicative linear functionals on $B$ are just the evaluations at points of $R$. Let $\mu_{t}$ be the functional defined by $\mu_{t}(f)=f(t)$. An easy computation shows that, for a finite $\operatorname{sum} \Sigma a_{k} \mu_{t_{k}}, \| \Sigma a_{k} \mu_{t_{k}}||=\Sigma\left|a_{k}\right|$. Let $\mathfrak{Y}_{0}$ be the subset of $\mathfrak{Y}$ consisting of these finite sums; $\mathfrak{Y}_{0}$ is dense in $\mathfrak{Y}$. For $f \in B$, the norm of $\alpha \pi(f)$ is the same as the norm of $\pi(f)$ as a functional over $\mathfrak{Y}$ and hence as functional over $\mathfrak{Y}_{0}$. Therefore $\|\alpha \pi(f)\|=$ $\|f\|_{\infty}$. It follows that $\alpha \pi$ is not bi-continuous.

3.17. THEOREM. Let (S) be a locally compact abelian group. Then $L^{* *}(\mathfrak{S}) / \mathfrak{Y} \perp$ is isometrically isomorphic to the algebra of all regular Borel measures on the almost periodic compactification of (S), with multiplication taken as convolution.

Proof. It is an immediate consequence of the fundamental approximation theorem for almost periodic functions [8, p. 126] that when (8) is a locally compact abelian group, the subspace $\mathfrak{Y}$ can be identified with the collection of almost periodic functions on $\mathbb{B}$. Let $\mathfrak{M}$ be the almost periodic compactification of $\$ S[13$, p. 124, ff.], [7, p. 165 ff.], and let $\alpha$ be the associated homomorphism of $\mathbb{S}$ onto a dense subset of $\mathfrak{M}$. Then $\alpha$ induces an algebraic isomorphism $\gamma$ of the complex valued continuous functions $\mathbb{C}(\mathfrak{M})$ onto the almost periodic functions on $\mathbb{S}$, which we identify with $\mathfrak{Y}$, by the following rule: $\gamma(f)(t)=f(\alpha(t)), t \in \mathbb{S}, f \in C(\mathfrak{M})$. Since the sup norm is involved when $\mathfrak{Y}$ is identified as a Banach algebra consisting of the almost periodic functions on $\mathbb{B}$, and since $\mathbb{B}$ is dense in $\mathfrak{M}$, it is clear that $\gamma$ is an isometry. Also since [13] $\gamma$ is an isomorphism of $C(\mathfrak{M})$ onto $\mathfrak{Y}$, its adjoint mapping $\gamma^{*}$ is a linear space isometric isomorphism of $\mathfrak{Y}^{*}$ onto $(C(\mathfrak{M}))^{*}$. As usual we may consider $(C(\mathfrak{M}))^{*}$ as an algebra, where the elements are regular Borel measures and 
multiplication is convolution [5, p. 265] [10, p. 229].

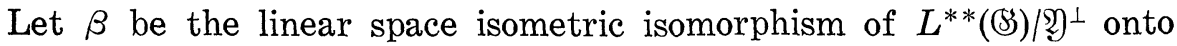
$\mathfrak{Y}^{*}$ defined by $\beta(F+\mathfrak{Y} \perp)=F \mid \mathfrak{Y}$, the restriction of $F$ to $\mathfrak{Y}$. The mapping $\gamma^{*} \beta$ is taken an isometric linear space isomorphism of $\left.L^{* *}(\mathbb{S}) / \mathfrak{Y}\right)^{\perp}$ onto $(C(\mathfrak{M}))^{*}$, and the proof reduces to showing that $\gamma^{*} \beta$ is an algebra isomorphism. Since the range of $\gamma^{*} \beta$ is $(C(\mathfrak{M}))^{*}$ it is sufficient to show that $\gamma^{*} \beta\left(\left(F+\mathfrak{Y}^{\perp}\right)\left(G+\mathfrak{Y}^{\perp}\right)\right)$ and $\gamma^{*} \beta\left(F+\mathfrak{Y}^{\perp}\right) \gamma^{*} \beta\left(G+\mathfrak{Y}^{\perp}\right)$ have the same Fourier-Stieltjes transform for each $F, G \in B^{* *}$. Let $g \in C(\mathfrak{M})$ be an arbitrary character of $\mathfrak{M}$. From the definition of $\gamma, \gamma g$ is a character of 83 . Thus, by Lemma 3.6,

$$
\begin{array}{r}
\gamma^{*} \beta\left(\left(F+\mathfrak{Y}^{\perp}\right)(G+\mathfrak{Y} \perp)\right)(g)=\beta\left(F G+\mathfrak{Y}^{\perp}\right)(\gamma g)=F G(\gamma g)=F(\gamma g) G(\gamma g) \\
=\gamma^{*} \beta\left(F+\mathfrak{Y}^{\perp}\right)(g) \gamma^{*} \beta\left(G+\mathfrak{Y}^{\perp}\right)(g)=\gamma^{*} \beta\left(F+\mathfrak{Y}^{\perp}\right) \gamma^{*} \beta\left(G+\mathfrak{Y}{ }^{\perp}\right)(g)
\end{array}
$$

and the desired relation holds.

A further identification of a quotient algebra of $L^{* *}(\mathbb{S})$ may be made relating the algebra $L^{* *}(\mathbb{S})$ to a familiar object study [10], namely the algebra of countably additive regular Borel measures on (S).

3.18. THeOREM. Let \&s be a locally compact abelian group. Let $\sqrt{5}$ be the subspace of $L^{*}(\mathbb{S})$ which may be identified with the continuous functions on (S) which vanish at infinity (or all continuous functions if $\mathbb{S}$ is compact). Then $\mathbb{S}^{\perp}$ is a two-sided ideal of $L^{* *}(\mathbb{S})$ and $L^{* *}(\mathbb{S}) / \mathbb{S}^{\perp}$ is isometrically isomorphic as an algebra to $M(\mathbb{S})$, the algebra of all countably additive regular Borel measures on $\mathbb{B}$.

Proof. If $\mathbb{B}$ is compact, the Peter-Weyl theorem yields $\mathfrak{C}=\mathfrak{Y}$, and $\mathbb{B}$ is its own almost periodic compactification so Theorem 3.17 yields the result. We therefore suppose that $\mathbb{B}$ is not compact. Let $f \in \mathbb{E}, x \in L(\mathbb{B})$. It was noted in the proof of Theorem 3.12 that $\langle f, x\rangle$ could be identified with the continous function on (B) whose value at $\beta \in \mathbb{B}$ is $\int f(\alpha+\beta) x(\alpha) d \alpha$. We show next that this function vanishes at infinity which will establish that $\langle f, x\rangle \varepsilon[$.

Let $\varepsilon>0$, and let $\Omega$ be a compact symmetric neighborhood of the identity $e$ of $\mathbb{S}$, such that $|f(\alpha)| \leqq \varepsilon / 2\|x\|$ if $\alpha \notin \Re$. Suppose $\mathfrak{U}$ is a compact symmetric neighborhood of $e$ such that $\int_{\mathfrak{u}}|x(\alpha)| d \alpha \geqq\|x\|-\varepsilon /(2\|f\|)$. Note that if $\beta \notin \mathfrak{R}+\mathfrak{U}$ and $\alpha \in \mathfrak{U}$ then $\alpha+\beta \notin \Re$ and $|f(\alpha+\beta)| \leqq$ $\varepsilon / 2\|x\|$. Then for $\beta \notin \Re+\mathfrak{U}$, a compact set,

$$
\begin{aligned}
\left|\int_{\mathscr{S}} f(\alpha+\beta) x(\alpha) d \alpha\right| & \leqq \int_{\mathfrak{u}}|f(\alpha+\beta)||x(\alpha)| d \alpha+\int_{\sim \mathfrak{l}}|f(\alpha+\beta)||x(\alpha)| d \alpha \\
& \leqq(\varepsilon / 2\|x\|) \int_{U}\left|x(\alpha) d \alpha+\|f\| \int_{\sim \mathfrak{n}}\right| x(\alpha) \mid d \alpha \leqq \varepsilon .
\end{aligned}
$$

Hence for $F \in \mathfrak{C}^{\perp}$ and $f \in \mathfrak{C},[F, f](x)=F(\langle f, x\rangle)=0, x \in L(\mathbb{S})$ and 
so $[F, f]=0$. Consequently for $G \in L^{* *}(\mathbb{S}), G F(f)=G([F, f])=0$, so $G F \in \mathbb{C}^{\perp}$. Thus $\mathbb{C}^{\perp}$ is a left ideal in $L^{* *}(\mathbb{S})$.

Suppose next that $f \in \mathbb{E}$ and $F \in L^{* *}(\$)$. Now $F$ restricted to $\mathbb{E}$ yields a functional on the collection of continuous functions on $\mathbb{B}$ vanishing at infinity, and as such is representable by a countably additive regular Borel measure $\nu$ on $\mathbb{B}$. We thus have

$$
F(g)=\int g(\alpha) d \nu(\alpha), g \in \mathfrak{C} .
$$

As we noted earlier, $\langle f, x\rangle \in \mathbb{E}$ if $f \in \mathbb{E}$ and $x \in L(\mathbb{S})$. Then if we use the representation of $\langle f, x\rangle$ as a function on $\mathbb{B}$, and use (3.12) we have

$$
\begin{aligned}
{[F, f](x) } & =F(\langle f, x\rangle)=\int_{\mathscr{S}}\langle f, x\rangle(\alpha) d \nu(\alpha)=\int_{\mathscr{S}}\left\{\int_{\mathscr{S}} f(\alpha+\beta) x(\beta) d \beta\right\} d \nu(\alpha) \\
& =\int_{\mathscr{S}}\left\{\int_{\mathscr{S}} f(\alpha+\beta) d \nu(\alpha)\right\} x(\beta) d \beta
\end{aligned}
$$

since both measures are completely additive regular Borel measures and the Fubini theorem applies. Consequently, $[F, f]$ may be identified with the function on $\&$ whose value at $\beta \in \mathbb{B}$ is $\int_{\mathscr{S}} f(\alpha+\beta) d \nu(\alpha)$, which function is clearly continuous on $\mathbb{S}$. We next show that the above function vanishes at infinity.

Let $\varepsilon>0$ be given. Let $\Re$ be a symmetric compact neighborhood of $e$ such that $|f(\alpha)| \leqq \varepsilon / 2\|\nu\|$, if $\alpha \notin \Re$. Suppose that $\mathfrak{U}$ is a compact symmetric neighborhood of $e$ such that $\int_{\mathfrak{u}}|d \nu(\alpha)| \geqq\|\nu\|-\varepsilon / 2\|f\|$. Then, for $\beta \notin \Re+\mathfrak{U}$,

$$
\begin{aligned}
\left|\int_{\mathscr{S}} f(\alpha+\beta) d \nu(\alpha)\right| & \leqq \int_{\mathfrak{u}}|f(\alpha+\beta)||d \nu(\beta)|+\int_{-\mathfrak{u}}|f(\alpha+\beta)||d \nu(\beta)| \\
& \leqq \varepsilon / 2\|\nu\| \int_{\mathfrak{u}}|d \nu(\beta)|+\|f\| \int_{\sim \mathfrak{u}}|d \nu(\beta)| \leqq \varepsilon .
\end{aligned}
$$

Hence $[F, f] \in \mathbb{F}$ whenever $f \in \mathbb{E}$. Thus if $F \in \mathbb{C}^{\perp}, G \in L^{* *}(\mathbb{S})$ and $f \in \mathfrak{C}$, $F G(f)=F([G, f])=0$, so $F G \in \mathbb{C}^{\perp}$. Consequently $\mathbb{C}^{\perp}$ is a right ideal of $L^{* *}(\$)$, and thus is a two sided ideal. From Banach space theory, there is an isometric linear space isomorphism between $L^{* *}(\mathbb{S}) / \mathbb{C}^{\perp}$ and $\mathbb{C}^{*}$. Also under the identification noted earlier $\mathbb{C}^{*}$ is isometrically isomorphic as a linear space to $M(\mathbb{S})$. The composite isometric isomorphism $\lambda$ is defined by $\lambda\left(F+\mathfrak{\Im}^{\perp}\right)=\nu$, where $F(f)=\int f(\alpha) d \nu(\alpha)$ for all $f \in \mathfrak{c}$.

It remains for us to see that $\lambda$ is an algebra isomorphism when $L^{* *}(\Omega) / \mathcal{\complement}^{\perp}$ is given the quotient space multiplication induced from the multiplication in $L^{* *}(\mathbb{S})$ and multiplication in $M(\mathbb{S})$ is convolution.

Let $F_{i} \in L^{* *}(\mathbb{S})$ and let $\nu_{i}=\lambda\left(F_{i}+\mathbb{S}^{\perp}\right), i=1,2$. Then let 


$$
\begin{gathered}
\lambda\left(\left(F_{1}+\left(\mathfrak{\mho}^{\perp}\right)\left(F_{2}+\sqrt{ }\right)\right)=\lambda\left(F_{1} F_{2}+\sqrt{5}\right)=\nu_{3} . \quad \text { Whence for all } f \in \mathfrak{\Im},\right. \\
\int f(\alpha) d \nu_{3}=F_{1} F_{2}(f)=F_{1}\left(\left[F_{2}, f\right]\right)=\int\left[F_{2}, f\right](\alpha) d \nu_{1}(\alpha),
\end{gathered}
$$

since as noted earlier $\left[F_{2}, f\right] \in \mathfrak{C}$. But

$$
\int\left[F_{2}, f\right](\alpha) d \nu_{1}(\alpha)=\int\left\{\int f(\beta+\alpha) d \nu_{2}(\beta)\right\} d \nu_{1}(\alpha)=\int f(\alpha) d \nu_{1} * \nu_{2}(\alpha) .
$$

Since $f$ ranges over all continuous functions vanishing at infinity $\nu_{3}=$ $\nu_{1} * \nu_{2}$ and $\lambda\left(\left(G_{1}+\left(^{\perp}\right) F_{2}+\left(^{\perp}\right)\right)=\lambda\left(F_{1}+{ }^{\perp}\right) * \lambda\left(F_{2}+\left(\mathcal{S}^{\perp}\right)\right.\right.$.

We are now in a position to show that the equality $\mathfrak{Y}^{\perp}=\mathfrak{R}^{* *}$ which held for a compact abelian group fails in the case of an abelian group which is not compact.

3.19. Lemma. Let \& be an abelian locally compact group that is not compact. Then $\Re^{* *} \subset \mathfrak{Y}^{\perp}$ and the inclusion is proper.

Proof. It follows from Theorem 3.7 that $\Re^{* *} \subset \mathfrak{Y}^{\perp}$. Consider first the case when $(S)$ is not discrete. Suppose $\Re^{* *}=\mathfrak{Y}^{\perp}$. As above let $(\mathfrak{S}$ be the subspace of $L^{*}(\mathbb{S})$ generated by those elements which may be identified with continuous functions on $(5)$ which vanish at infinity. It follows from Theorem 3.18 that $\Re^{* *} \subset \mathfrak{C}^{\perp}$. Thus $\mathfrak{Y}^{\perp} \subset \mathfrak{C}^{\perp}$, and consequently $\mathfrak{S} \subset \mathfrak{Y}$. As noted in the proof of Theorem 3.18, $\mathfrak{Y}$ can be identified with the collection of almost periodic functions on $\mathbb{B}$. However, since $\mathbb{B S}$ is not compact, $\mathbb{C} \cap \mathfrak{Y}=(0)$ is a consequence of the theory of almost periodic functions on groups.

For suppose $f \neq 0, f \in \mathfrak{Y} \cap \mathbb{E}$. Pick $\varepsilon>0$ so that $\left|f\left(\alpha_{0}\right)\right|>3 \varepsilon$ and $|f(\alpha)|<\varepsilon$, for $\alpha \notin \Re$, for some compact set $\Re$. There is [13, p. 133] a finite set $\beta_{i}, i=1, \cdots, n$, in (B) so that for any $\gamma \in \mathbb{B}$, there is an integer $j, 1 \leqq j \leqq n$ such that $\left|f\left(\gamma^{-1} \alpha\right)-f\left(\beta_{i}^{-1} \alpha\right)\right|<\varepsilon$, for all $\alpha \in \mathbb{B}$. Thus for arbitrary $\gamma$, we may pick $\alpha=\gamma \alpha_{0}$, and $\left|f\left(\alpha_{0}\right)-f\left(\beta_{j}^{-1} \gamma \alpha_{0}\right)\right|<\varepsilon$ for arbitrary $\gamma$, for some $j, 1 \leqq j \leqq n$. In particular if $\gamma$ is taken so that $\gamma \notin \beta_{j} \Re \alpha_{0}^{-1}, j=1, \cdots, n$, as is possible since $(S)$ is not compact, we have a contradiction. Thus $\mathfrak{C} \subset \mathfrak{Y}$ is impossible and the proof of the lemma is complete.

3.20. Lemma. Let (\$3 be a locally compact abelian group. Then each of the algebras $L^{* *}(\mathbb{S}) / \Re^{* *}$ and $L^{* *}(\mathbb{S}) / \mathfrak{Y}^{\perp}$ has an identity.

Proof. Since, by the second isomorphism theorem for rings, $\left.L^{* *}(\mathbb{S}) / \mathfrak{Y}\right)^{\perp}$ is a homomorphic image of $L^{* *}(\mathbb{S}) / \Re^{* *}$, it suffices to show that $L^{* *}(\mathbb{S}) / \Re^{* *}$ has an identity. Now $L(\mathbb{S})$ has an approximate identity, so by Lemma $3.8, L^{* *}(\$)$ has a right identity $E$. It is immediate that $E+\mathfrak{R}^{* *}$ is

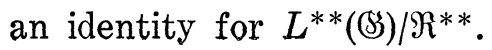


3.21. TheOREM. Let (S) be a locally compact abelian group. Then the algebra $L^{* *}(\mathbb{S}) / \Re^{* *} \cong L(\mathbb{S})$ if and only if $\mathbb{B}$ is a finite group.

Proof. If $\mathbb{B S}$ is a finite group $L(\mathbb{B})$ is a finite-dimensional Banach space so $L^{* *}(\$) \cong L(\$)$ and $\Re^{* *}=(0)$.

Suppose $\mathbb{B S}$ is not discrete. Then by Lemma $3.20, L^{* *}(\mathbb{S}) / \Re^{* *}$ has an identity while $L(\mathbb{S})$ does not so $L^{* *}(\mathbb{S}) / \Re^{* *} \cong L(\mathbb{S})$ is impossible. Suppose finally that $(\mathbb{S}$ is infinite and discrete. We saw in Theorem 3.3 that, for an infinite discrete abelian group (S), $L^{* *}(\mathbb{S}) / \Re^{* *}$ could be written as the direct sum of two ideals $\widetilde{\Im}_{1}$ and $\widetilde{\Im}_{2}$ where $\widetilde{\Im}_{1}$ is a maximal ideal and $\Im_{2}$ is the set of all scalar multiples of an idempotent. Suppose we had $L(\mathfrak{S}) \cong L^{* *}(\mathbb{S}) / \mathfrak{R}^{* *}$. Then we would have $L(\mathbb{S})=\mathfrak{M} \oplus \Im$ where $\mathfrak{I}$ is the set of scalar multiples of an element $z \neq 0$ which annihilates $\mathfrak{M}$. Since a closed ideal in $L(\mathbb{S})$ is invariant under translation, there is a complex function $\lambda$ on $\$$ s such that $z(\alpha+\beta)=\lambda(\alpha) z(\beta)$ for all $\alpha, \beta \in \mathbb{B}$. It is immediate that $\lambda$ is a homomorphism of $B$ into the complexes. As $z \neq 0, \lambda$ is bounded, so is a character on 8 S. Thus $|\lambda(\alpha)|=1, \alpha \in G$. Hence if $z(\beta) \neq 0,\|z\|=\sum_{\alpha \in \mathbb{S}}|z(\alpha+\beta)|=\sum_{\alpha \in \mathscr{G}}|\lambda(\alpha)||z(\beta)|$ is possible only if $\mathbb{S}$ is a finite group. Thus $L^{* *}(\mathbb{S}) / \Re^{* *}=L(\mathbb{S})$ is impossible for (S) an infinite discrete abelian group.

3.22. THeOREM. Let (S) be a locally compact abelian group. Then $\left.L^{* *}(\mathbb{S}) / \mathfrak{Y}\right)^{\perp} \cong L(\mathbb{S})$ if and only if (S) is finite.

Proof. If $\mathbb{B S}$ is finite $L(\mathbb{S})$ is reflexive and $\mathfrak{Y}=L^{*}(\mathbb{S})$ by the PeterWeyl theorem so the stated isomorphism holds.

If $(B)$ is not a discrete group, then $L(\mathbb{S})$ has no identity, while Lemma 3.20 gives an identity for $\left.L^{* *}(\mathbb{S}) / \mathfrak{Y}\right)^{\perp}$, so there can be no isomorphism.

Suppose that $(S)$ is a discrete group and $L^{* *}(\mathbb{S}) / \mathfrak{Y}^{\perp} \cong L(\mathbb{S})$. Theorem 3.17 yields a compact group $\mathfrak{K}$, the almost periodic compactification of (S), such that $\left.L^{* *}(\mathbb{S}) / \mathfrak{Y}\right)^{\perp} \cong M(\mathfrak{S})$, the algebra under convolution of the regular Borel measures on $\mathfrak{S}$. Thus $L(\mathbb{S}) \cong M(\mathfrak{C})$. Let $\Delta$ be the character group of $\$ S$ and let $\Gamma$ be the character group of $\mathfrak{S}$. Since $L(\mathbb{S}) \cong$ $M(\mathfrak{S})$ we may identify the maximal ideal space of $M(\mathfrak{E})$ as $\Delta$, and the topologies coincide in the two interpretations. Now $\Gamma$ may be interpreted as a subset of the maximal ideal space of $M(\mathfrak{2})$, and thus as a subset of 4 , and the topology of $\Gamma$ as a topological group coincides [10, p. 232] with the topology as a subset of $\Delta$. Also [10, p. 235] $\Gamma$ is an open subset of $\Delta$. As a topological group $\Gamma$ is discrete, since $\mathfrak{S}$ is compact. As a topological group $\Gamma$ is discrete, since $\mathfrak{S}$ is compact. Thus the points of $\Gamma$ are open sets in $\Delta$. But $\Delta$ is a compact topological group, since $(B)$ is discrete. Since $\Delta$ has an open set consisting of a point, $\Delta$ is a discrete group. Therefore, being both compact and discrete, $\Delta$ is a finite group and so (B) is a finite group. 
4. A further example. The examples given in $\S 3$ were principally of the nature that the given Banach algebra $B$ was commutative and semi-simple, while the algebra $B^{* *}$ was not commutative and not semisimple. The present section is concerned with an example in which the Banach algebra $B$ is commutative and semi-simple, while the algebra $B^{* *}$ is commutative but not semi-simple.

Let $B$ be a Banach space, and let $₹$ be a total subspace of $B^{*}$. We denote by $\sigma(B, \mathfrak{F})$ the weak topology on $B$ determined by the subspace $\mathfrak{F}$, that is, the smallest topology on $B$ which makes all the members of $\mathfrak{F}$ continuous. Similarly, we use the notation $\sigma\left(B^{* *}, \mathfrak{F}\right)$ for the corresponding topology in $B^{* *}$.

4.1. Theorem. Let $B$ be Banach space, and let $\mathfrak{F}$ be a closed subspace of $B^{*}$ which is total. Then a necessary and sufficient condition that $\pi(B) \oplus \mathfrak{F}^{\perp}=B^{* *}$ is that the unit ball $S$ of $B$ be relatively compact with respect to the topology $\sigma(B, \mathfrak{F})$. The above decomposition can occur only if $B$ is isomorphic to conjugate space.

This is a slightly more explicit form of a part of Theorem 17' of Dixmier [4, p. 1069]. Since the same arguments used by Dixmier give the present version, we omit the details.

4.2. THEOREM. There exists a semi-simple commutative Banach algebra, for which the algebra $B^{* *}$ is commutative but not semi-simple.

Proof. Let $B=l_{1}$, the space of absolutely convergent sequences of complex numbers, with its usual norm, and let multiplication in $B$ be defined coordinatewise, i.e. $\left\{a_{k}\right\}\left\{b_{k}\right\}=\left\{a_{k} b_{k}\right\}$. It is readily verified that $B$ is a complex commuatative semi-simple Banach algebra. It is also readily verified that any multiplicative linear functional $f$ is of the form $f\left(\left\{a_{k}\right\}\right)=a_{m}$ for some integer $m$. Let $\mathfrak{Y}$ be the closed subspace of $B^{*}$ generated by the multiplicative linear functionals. Note that $B^{*}$ may be identified with $(m)$, the space of all bounded sequences with the sup norm. Under this identification $\mathfrak{g}$ becomes the closure in $(m)$ of the collection of all sequence with a finite number of non-zero terms, i.e. $\mathfrak{Y}$ may be identified with $\left(c_{0}\right)$, considered as a subspace of $(m)$. The topology $\sigma(B, \mathfrak{Y})$ is then the same topology on $B$ as its $w^{*}$-topology when $B$ is considered as the conjugate space of $\left(c_{0}\right)$. Since the space $Y$ is total, and since Alaoglu's theorem asserts that the unit ball of $B$ is compact in $\sigma(B, \mathfrak{Y})$, Theorem 4.1 asserts that $\left.B^{* *}=\pi(B) \oplus \mathfrak{Y}\right)^{\perp}$.

We next show that condition (i) of Theorem 3.10 is satisfied. For our calculations we make the identifications indicated above. Let $f \in B^{*}$ and $x \in B$. Say $f=\left\{c_{k}\right\} \in(m)$ and let $x=\left\{x_{k}\right\}, y=\left\{y_{k}\right\}$ be in $l_{1}$. Then $\langle f, x\rangle(y)=f(x y)=\sum_{k} c_{k} x_{k} y_{k}$. Thus, in $(m),\langle f, x\rangle=\left\{c_{k} x_{k}\right\}$. However, 
as $\left\{x_{k}\right\} \in l_{1}$ and $\left\{c_{k}\right\} \in(m),\left\{c_{k} x_{k}\right\} \in\left(c_{0}\right)$ and so $\langle f, x\rangle \in \mathfrak{Y}$.

Theorem 3.10 then asserts that $B^{* *} \mathfrak{Y}^{\perp}=(0)$ and $\mathfrak{Y}^{\perp} \pi(B)=(0)$, and Corollary 3.11 asserts that $\mathfrak{Y}^{\perp}$ is the radical of $B^{* *}$. The relation $B^{* *}=\pi(B) \oplus \mathfrak{Y}^{\perp}$ together with the above comment shows that multiplication is commutative in $B^{* *}$. Furthermore, since $B$ is not reflexive, $B^{* *}$ is not semi-simple.

In the foregoing example, the relationships $B^{* *}=\pi B \oplus \mathfrak{Y}^{\perp}=\pi B \oplus \mathfrak{R}^{* *}$ were observed. This is in contrast to the situation noted in $\S 3$, where we saw in Theorems 3.31 and 3.32 that for an infinite locally compact abelian group one could not even have either $L^{* *}(\mathbb{S}) / \Re^{* *} \cong L(\mathbb{S})$ or $\left.L^{* *}(\mathfrak{S}) / \mathfrak{Y}\right)^{\perp} \cong L(\mathfrak{S})$.

5. On the embedding of ideals. We consider here what happens to ideals in $B$ under the natural embedding $\pi$ of $B$ into $B^{* *}$. Recall that multiplication $F G$ in $B^{* *}$ is $w^{*}$-continuous in $F$ if $G$ is fixed and that $\pi(x) G$ is $w^{*}$-continuous in $G$ if $x \in B$ is held fixed.

5.1. Lemma. Let $\Im_{r}\left(\Im_{l}\right)$ be a right (left) ideal of $B$. Let $\Re_{r}\left(\Re_{l}\right)$ be the $w^{*}$-closure of $\pi\left(\Im_{r}\right)\left(\pi\left(\Im_{l}\right)\right)$. Then $\Re_{r}\left(\Re_{l}\right)$ is a right (left) ideal of $B^{* *}$.

Proof. Let $F \in \mathfrak{\Re}_{r}, F=w^{*}-\lim \pi\left(x_{a}\right), x_{\alpha} \in \Im_{r} . \quad$ Let $G \in B^{* *}, G=$ $w^{*}-\lim \pi\left(y_{\beta}\right), y_{\beta} \in B$. Then $F G=w^{*}-\lim \pi\left(x_{\alpha}\right) G$. Hence $F G \in \Re_{r}$ if we can show that $\pi\left(x_{\alpha}\right) G \in \mathfrak{R}_{r}$ for each index $\alpha$. But $\pi\left(x_{\alpha}\right) G=w^{*}-\lim \pi\left(x_{\alpha}\right) \pi\left(y_{\beta}\right)$. However $x_{\alpha} y_{\beta} \in \Im_{r}$ so that $\pi\left(x_{\alpha}\right) G \in \Re_{r}$. Let $F \in \Re_{l}$ and use the same notation. Then $\pi\left(y_{\beta}\right) F=w^{*}-\lim \pi\left(y_{\beta}\right) \pi\left(x_{a}\right) \in \Re_{l}$ and hence $G F \in \Re_{l}$.

\subsection{THEOREM.}

(a) If $\mathfrak{\Im}_{r}\left(\mathfrak{\Im}_{l}\right)$ is a proper regular right (left) ideal of $B$ then the $w^{*}$-closure of $\pi\left(\mathfrak{\Im}_{r}\right)\left(\mathfrak{\Im}_{l}\right)$ ) is a proper regular right (left) ideal of $B^{* *}$.

(b) If $\mathfrak{P}$ is a primitive ideal of $B$ then the $w^{*}$-closure of $\pi(\mathfrak{P})$ is contained in a primitive ideal of $B^{* *}$.

(c) If $\mathfrak{\Im}$ is a nilpotent right (left) ideal of $B$, then the $w^{*}$-closure of $\pi(\Im)$ is a nilpotent right (left) ideal of $B^{* *}$.

Proof. Let $\Re_{r}\left(\Re_{l}\right)$ be the indicated $w^{*}$-closure in (a). By Lemma 5.1, $\Re_{r}\left(\Re_{l}\right)$ is a right (left) ideal of $B^{* *}$. Let $j$ be a left (right) identity for $B$ modulo $\Im_{r}\left(\Im_{l}\right)$. We show that $\pi(j) \notin \Re_{r}\left(\Re_{l}\right)$. Since $\Im_{r}$ is a proper right ideal, dist $\left(j, \mathfrak{\Im}_{r}\right) \geqq 1$. Let $f \in B^{*}, f(j)=1$ and $f\left(\Im_{r}\right)=0$. Then $\pi(x)(f)=0, x \in \mathfrak{\Im}_{r}$ and $F(f)=0, F \in \mathfrak{\Re}_{r}$, while $\pi(j)(f)=1$.

Consider the $\mathfrak{\Im}_{r}$ case, where $\pi(j) \pi(x)-\pi(x) \in \pi\left(\mathfrak{\Im}_{r}\right)$ for all $x \in B$. Thus $\pi(j) F-F \in \Omega_{r}$ for all $F \in B^{* *}$. Hence $\pi(j)$ is a left identity for $B^{* *}$ modulo $\Omega_{r}$. 
Let $\mathfrak{P}$ be primitive ideal. We can write $\mathfrak{P}=(\mathfrak{M}: B)=\{x \in B \mid y x \in \mathfrak{M}$ for all $y \in B\}$ where $\mathfrak{M}$ is a regular maximal right ideal of $B$. Let $\Re$ be the $w^{*}$-closure of $\pi(\mathfrak{M})$ and let $\mathfrak{D}$ be the $w^{*}$-closure of $\pi(\mathfrak{P})$. Now $\pi(y) \pi(x) \in \Re$ for all $y \in B, x \in \mathfrak{P}$. Thus $\pi(y) F \in \Re$ for all $y \in B, F \in \mathfrak{Q}$ and then $G F \in \Re$ for all $G \in B^{* *}, F \in \mathfrak{Q}$. By (a), $\Re$ is contained in a regular maximal right ideal $\mathfrak{B}$ of $B^{* *}$ so that $\mathfrak{D} \subset\left(\mathfrak{B}: B^{* *}\right)$. This completes the proof of (b).

Let $\Im$ be a nilpotent right ideal of $B$, $\mathfrak{\Im}^{n}=(0)$. Let $\Re$ be the $w^{*}$-closure of $\pi(\Im)$ and $F_{k} \in \Re, k=1, \cdots, n$. We show $F_{1} F_{2} \cdots F_{n}=0$. Let $F_{n}=w^{*}-\lim \pi\left(x_{\alpha}\right), x_{\alpha} \in \mathfrak{\Im}$. For any $x_{1}, \cdots, x_{n-1} \in \mathfrak{\Im}, 0=\pi\left(x_{1}\right) \cdots \pi\left(x_{n-1}\right) \pi\left(x_{\alpha}\right)$ converges $\left(w^{*}\right)$ to $0=\pi\left(x_{1}\right) \cdots \pi\left(x_{n-1}\right) F_{n}$. Let $F_{n-1}=w^{*} \lim \pi\left(x_{\beta}\right)$. Then $\pi\left(x_{1}\right) \cdots \pi\left(x_{n-2}\right) \pi\left(x_{\beta}\right)$ converges $\left(w^{*}\right)$ to $\pi\left(x_{1}\right) \cdots \pi\left(x_{n-2}\right) F_{n-1}$ so that $0=$ $\pi\left(x_{1}\right) \cdots \pi\left(x_{n-2}\right) F_{n-1} F_{n}$. We can continue in this fashion to obtain $F_{1} F_{2} \cdots F_{n}=0$.

In the commutative case we can be more specific.

5.3. Theorem. Let $B$ be a complex commutative Banach algebra and $M$ be a regular maximal ideal of $B$. Then the $w^{*}$-closure $\Omega$ of $\pi(M)$ is a regular maximal two-sided ideal of $B^{* *}$.

Proof. There exists a multiplicative linear functional $f \in B^{*}$ such that $M=\{x \in B \mid f(x)=0\}$. Let $\mathfrak{B}=\left\{F \in B^{* *} \mid F(f)=0\right\}$. Clearly $\Re \subset \mathfrak{B}$. Let $j$ be an identity for $B$ modulo $M$. Now the smallest linear manifold containing $M$ and $j$ is $B$. Thus the linear manifold $\&$ in $B^{* *}$ generated by $\Re$ and $\pi(j)$ contains $\pi(B)$. However $\Re$ is $w^{*}$-closed and $\mathbb{L}$ is the direct sum of $\Omega$ and the one-dimensional space generated by $\pi(j)$. Therefore $\mathfrak{Q}$ is $w^{*}$-closed. By Helly's theorem, $\pi(B)$ is $w^{*}$-dense in $B^{* *}$ so that $\mathbb{R}=B^{* *}$. It follows that $\Omega$ is a maximal closed linear manifold of $B^{* *}$ and that $\Re=\mathfrak{W}$. In view of Lemma $3.6, \Re$ is a maximal twosided ideal of $B^{* *}$.

5.4. Lemma. If $e$ is an identity for $B$ then $\pi(e)$ is an identity for $B^{* *}$.

Proof. One verifies that, for $f \in B^{*},\langle f, e\rangle=f=[\pi(e), f]$. From this it readily follows that $\pi(e) F=F^{i} \pi(e)=F$ for every $F \in B^{* *}$.

Suppose now that $B^{* *}$ is commutative and $B$ has an identity. By Lemma 5.4, so does $B^{* *}$. Let $\mathfrak{M}\left(\mathfrak{M}_{2}\right)$ be the space of maximal ideals of $B\left(B^{* *}\right)$. Each $M_{2} \in \mathfrak{M}_{2}$ determines a unique maximal ideal $\pi^{*}\left(M_{2}\right)$ of $B$ by the rule $x\left[\pi^{*}\left(M_{2}\right)\right]=\pi(x)\left(M_{2}\right), x \in B$. This is the "adjoint transformation" of $\mathfrak{M}_{2}$ into $\mathfrak{M}$ induced by the mapping $\pi$ of $B$ into $B^{* *}$ (see $\left[7\right.$, p. 76]). As shown there, $\pi^{*}$ is continuous.

5.5. Theorem. Let $B^{* *}$ be commutative and $B$ contain an identity. 
Then $\pi^{*}$ maps $\mathfrak{M}_{2}$ continuously onto $\mathfrak{M} ; \pi^{*}$ is a homeomorphism if and only if every maximal ideal of $B^{* *}$ is $w^{*}$-closed.

Proof. Let $M \in \mathfrak{M}$. By Theorem 5.3, the $w^{*}$-closure of $\pi(M)$ is an element $M_{2} \in \mathfrak{M}_{2}$ and it is clear that $\pi^{*}\left(M_{2}\right)=M$. Thus $\pi^{*}$ maps $\mathfrak{M}_{2}$ onto $\mathfrak{M}$. Let $\mathfrak{M}_{2}$ be the set of $M_{2} \in \mathfrak{M}_{2}$ which are $w^{*}$-closed. Since $\pi^{*}\left(\mathfrak{W}_{2}\right)=\mathfrak{M}$, we have $\mathfrak{W}_{2}=\mathfrak{M}_{2}$ if $\pi^{*}$ is one-to-one. Suppose that $\mathfrak{M}_{2}=\mathfrak{M}_{2}$. Let $M_{2}$ and $M_{2}^{\prime} \in \mathfrak{M}_{2}$ where $\pi^{*}\left(M_{2}\right)=\pi^{*}\left(M_{2}^{\prime}\right)$. Clearly $M_{2} \supset$ $\pi\left(\pi^{*}\left(M_{2}\right)\right)$ so that the $w^{*}$-closure of the latter set is, by Theorem 5.3, $M_{2}$. Hence $M_{2}=M_{2}^{\prime}$.

That $\pi^{*}$ can be a homeomorphism in the case of a non-trivial algebra $B$ is seen from the example of $\S 4$ (adjoin an identity to that $B$ ). In general $\pi^{*}$ is not one-to-one. For a case in point consider $B=C(X)$ where $X$ is compact. It is known that $B^{* *}$ is of the form $C(Y)$ where $Y$ is compact (see [1, Theorem 4.8] or $\S 7$ ). Suppose that $\pi^{*}$ is one-toone. From the relation $x\left(\pi^{*}\left(M_{2}\right)\right)=\pi(x)\left(M_{2}\right)$ it follows that $\pi(B)$ contains all continuous functions on $\mathfrak{M}_{2}$ or $\pi(B)=B^{* *}$. But this implies that $X$ is a finite set.

5.6. THEOREM. Let $\Re\left(\Re^{* *}\right)$ be the radical of $B\left(B^{* *}\right)$. Then $\pi(\Re)=$ $\Re^{* *} \cap \pi(B)$ if $B$ is commutative or satisfies the descending chain condition for left ideals.

We believe this theorem true for any Banach algebra $B$. First we have a preliminary result.

5.7. Lemma. An element $x \in B$ is left (right) quasi-regular if and only if $\pi(x)$ is left (right) quasi-regular in $B^{* *}$.

Proof. Let $L_{x}\left(R_{x}\right)$ be the operation on $B$ of left (right) multiplication by $x$ and let $I$ be the identity operator acting on $B$. For $F \in B^{* *}$ let $L_{F}\left(R_{F}\right)$ be similarly defined. Note that $\langle f, x\rangle(y)=f\left(L_{x}(y)\right)$ so that $\langle f, x\rangle=L_{x}^{*}(f), f \in B^{*}, x, y \in B$. Then, for $F \in B^{* *}, \pi(x) F(f)=[F, f](x)=$ $F(\langle f, x\rangle)=L_{x}^{* *} F(f)$. Hence $\pi(x) F=L_{x}^{* *}(F)$. A similar argument shows that $F \pi(x)=R_{x}^{* *}(F)$.

Suppose that $\pi(x)$ is right quasi-regular in $B^{* *}$ where $\pi(x)+F-$ $\pi(x) F=0$. For any $G \in B^{* *}$ we have $G-(\pi(x)+F-\pi(x) F) G=G$ which can be rewritten as

$$
\left(I^{* *}-L_{x}^{* *}\right)\left(I^{* *}-L_{F}\right)(G)=G .
$$

It follows that $I^{* *}-L_{x}^{* *}$ is a mapping of $B^{* *}$ onto $B^{* *}$. By the theory of linear operators $I-L_{x}$ maps $B$ onto $B$. Hence there is exists $y \in B$ such that $\left(I-L_{x}\right) y=-x$ or $x+y-x y=0$.

Suppose that $\pi(x)$ is left quasi-regular where $\pi(x)+F-F \pi(x)=0$. Arguing as before we have 


$$
\left(I^{* *}-R_{x}^{* *}\right)\left(I^{* *}-R_{F}\right)(G)=G
$$

and can show that $x$ is left quasi-regular.

It follows that, for $x \in B$, the spectrum of $x$ as an element of $B$ is the same as that of $\pi(x)$ as an element of $B^{* *}$. Moreover $x$ is in the radical of $B$ if $\pi(x)$ lies in the radical of $B^{* *}$.

We turn to the proof of Theorem 5.6. Suppose that $B$ is commutative and $x \in \Re$. By Lemma 3.9, $\pi(x)$ lies in the center of $B^{* *}$. Let $\rho(G)$ denote the spectral radius of an element $G \in B^{* *}$. Since $\pi(x)$ permutes with $G, \rho(\pi(x) G) \leqq \rho(\pi(x)) \rho(G)=0$. It follows that $\pi(x) G$ is quasi-regular for all $G \in B^{* *}$ whence $\pi(x) \in \Re^{* *}$. Next suppose that $B$ satisfies the descending chain condition on left ideals and $x \in \Re$. Then $x$ lies in a nilpotent left ideal. It follows from Theorem 5.2 that $\pi(x) \in \Re^{* *}$. By Lemma 5.7, $\pi(\Re) \supset \Re^{* *} \cap \pi(B)$.

6. Extension of mappings. Let $B_{1}$ and $B_{2}$ be two Banach algebras. It turns out that if $T$ is a continuous homomorphism of $B_{1}$ into $B_{2}$ then $T^{* *}$ is a homomorphism of $B_{1}^{* *}$ into $B_{2}^{* *}$. This phenomenon fails, in general, for anti-homomorphisms.

6.1. Theorem. Let $B_{1}$ and $B_{2}$ be two Banach algebras. Let $T$ be a continuous homomorphism of $B_{1}$ into $B_{2}$. Then $T^{* *} i s$ a homomorphism of $B_{1}^{* *}$ into $B_{2}^{* *}$.

Proof. Let $F, G \in B_{1}^{* *}$ and $g \in B_{2}^{*}$. We wish to show that $T^{* *}(F G)=$ $T^{* *}(F) T^{* *}(G)$. We have

$$
T^{* *}(F G)(g)=F G\left(T^{*}(g)\right)=F\left(\left[G, T^{*}(g)\right]\right)
$$

and

$$
T^{* *}(F) T^{* *}(G)(g)=T^{* *}(F)\left(\left[T^{* *}(G), g\right]\right)=F\left(T^{*}\left(\left[T^{* *}(G), g\right]\right)\right) .
$$

Thus the desired relation follows if we verify

$$
\left[G, T^{*}(g)\right]=T^{*}\left(\left[T^{* *}(G), g\right]\right)
$$

for all $G \in B_{1}^{* *}, g \in B_{2}^{*}$. Let $x \in B_{1}$. Now $\left[G, T^{*}(g)\right](x)=G\left(\left\langle T^{*}(g), x\right\rangle\right)$ and $T^{*}\left(\left[T^{* *}(G), g\right]\right)(x)=T^{* *}(G)(\langle g, T(x)\rangle)=G T^{*}(\langle g, T(x)\rangle)$. Therefore (4.3) holds if we can show

$$
\left\langle T^{*}(g), x\right\rangle=T^{*}(\langle g, T(x)\rangle)
$$

for all $g \in B_{2}^{*}, x \in B_{1}$. Let $y \in B_{1}$. Then $\left\langle T^{*}(g), x\right\rangle(y)=T^{*}(g)(x y)=$ $g(T(x y))$ while $T^{*}(\langle g, T(x)\rangle)(y)=g(T(x) T(y))$. Since $T$ is a homomorphism, (6.4) is valid.

6.2. THeorem. Let $T$ be a continuous anti-homomorphism of $B_{1}$ 
into $B_{2}$. Then $T^{* *}$ is an anti-homomorphism if and only if $F_{2} G_{2}=$ $G_{2} \cdot F_{2}$ for any pair $F_{2}, G_{2}$ in the range of $T^{* *}$.

Proof. For the notation see $\S 2$. Suppose that the $F_{2} G_{2}=G_{2} \cdot F_{2}$ condition holds. Let $F, G \in B_{1}^{* *}$ and $f \in B_{2}^{*}$. Then $T^{* *}(F G)(f)=$ $F([G, T f])$ while

$$
\begin{aligned}
T^{* *}(G) T^{* *}(F)(f) & =T^{* *}(F) \cdot T^{* *}(G)(f) \\
& =T^{* *}(F)\left(\left[T^{* *}(G) \mid f\right]\right)=F T^{*}\left(\left[T^{* *}(G) \mid f\right]\right) .
\end{aligned}
$$

To see that $T^{* *}$ is an anti-homomorphism it is sufficient then to show

$$
\left[G, T^{*}(f)\right]=T^{*}\left(\left[T^{* *}(G) \mid f\right]\right)
$$

for all $G \in B_{1}^{* *}, f \in B_{2}^{*}$. Let $x \in B_{1}$. We have $\left[G, T^{*}(f)\right](x)=$ $G\left(\left\langle T^{*}(f), x\right\rangle\right)$ while $T^{*}\left(\left[T^{* *}(G) \mid f\right]\right)(x)=T^{* *}(G)(\langle f \mid T(x)\rangle)$. Therefore to show (6.5) it is sufficient to show

$$
\left\langle T^{*}(f), x\right\rangle=T^{*}(\langle f \mid T(x)\rangle)
$$

for all $f \in B_{2}^{*}, \quad x \in B_{1}$. Let $y \in B_{1} . \quad\left\langle T^{*}(f), x\right\rangle(y)=f(T(x y))$ and $T^{*}(\langle f \mid T(x)\rangle)(y)=f(T(y) T(x))$. Since $T$ is an anti-homomorphism (6.7) is verified.

Assume that $T^{* *}$ is an anti-homomorphism. Suppose that there exists $F_{1}, G_{1} \in T^{* *}\left(B_{1}^{* *}\right)$ such that $F_{1} \cdot G_{1} \neq G_{1} F_{1}$. There exist $F, G \in B_{1}^{*}$ where $T^{* *}(F)=F_{1}, \quad T^{* *}(G)=G_{1}$ and, for some $f \in B_{2}^{*}, T^{* *}(F) \cdot T^{* *}$ $(G)(f) \neq T^{* *}(G) T^{* *}(F)(f)$. But $T^{* *}(F) \cdot T^{* *}(G)(f)=F\left(T^{*}\left[T^{* *}(G) \mid f\right]\right)$ while $T^{* *}(G) T^{* *}(F)(f)=T^{* *}(F G)(f)=F\left(\left[G, T^{*}(f)\right]\right.$. It follows that, for some $x \in B_{1}, T^{*}\left(\left[T^{* *}(G) \mid f\right]\right)(x) \neq\left[G, T^{*}(f)\right](x)$. However $T^{*}\left(\left[T^{* *}(G) \mid f\right]\right)$ $(x)=T^{* *}(G)(\langle f \mid T(x)\rangle)=G\left(T^{*}(\langle f \mid T(x)\rangle)\right)$ while $\left[G, T^{*}(f)\right](x)=G\left(\left\langle T^{*}\right.\right.$ $(f), x\rangle)$ so there must exist $y \in B_{1}$ where $T^{*}(\langle f \mid T(x)\rangle)(y) \neq\left\langle T^{*}(f)\right.$, $x\rangle(y)$. However these are equal, which completes the proof.

It is readily verified by the above technique that an involution on $B$ i.e. a conjugate linear anti-automorphism of period two, can be extended to an involution on $B^{* *}$ if the multiplication on $B$ is regular.

6.3. CoRollary. If $B_{1}$ is a closed subalgebra of the Banach algebra $B_{2}$ and the multiplication in $B_{2}$ is regular then so is that in $B_{1}$.

Proof. Let $T$ be the imbedding of $B_{1}$ into $B_{2}$. By Theorem 6.1, $T^{* *}(F G)=T^{* *}(F) T^{* *}(G)$ for $F, G \in B_{1}$. Now likewise $T^{* *}(G \cdot F)=$ $T^{* *}(G) \cdot T^{* *}(F)=T^{* *}(F) T^{* *}(G)$ since multiplication in $B_{2}$ is regular. Therefore $T^{* *}(G \cdot F)=T^{* *}(F G)$. Since $T^{* *}$ is one-to-one, the conclusion follows. 
Banach algebra $B_{1}$ onto the Banach algebra $B_{2}$ and if the multiplication in $B_{1}$ is regular then so is the multiplication in $B_{2}$.

Proof. By Theorem 6.1, $T^{* *}(F G)=T^{* *}(F) T^{* *}(G)$ for all $F, G \in B_{1}^{* *}$. If we give both $B_{1}$ and $B_{2}$ their transposed multiplication we see that $T^{* *}(G \cdot F)=T^{* *}(G) \cdot T^{* *}(F)$. But $T^{* *}(G \cdot F)=T^{* *}(F G)$. Since the range of $T^{* *}$ is all of $B_{2}$ the conclusion follows from Theorem 6.2.

7. On $B^{*}$-algebras. Let $B$ be a $B^{*}$-algebra. We show that the following theorem is contained implicitly in the work of Sherman and Takeda [12] on the second conjugate spaces of such $B$.

7.1. Theorem. Let $B$ be a $B^{*}$-algebra. Then $B^{* *}$ is a $B^{*}$-algebra in the Arens multiplication. The multiplication in $B$ is regular.

Proof. We know that $B$ is also a $C^{*}$-algebra [9, p. 281]. Following Takeda [12] we consider a canonical faithful ${ }^{*}$-representation $x \rightarrow x^{*}$ of $B$ as operators on a specially chosen Hilbert space $H$ where $H$ is the direct sum of Hilbert spaces $H_{f}$ defined for each state $f$. Let $B^{*}$ be the image of $B$ under this representation. Takeda shows that each $f \in B^{*}$ can be represented in the form

$$
f(x)=\sum_{k=1}^{n}\left(x^{\sharp}\left(\xi_{k}\right), \eta_{k}\right)
$$

where each $\xi_{k}, \eta_{k} \in H$. Conversely any functional of the form (7.1) must be bounded on $B$.

Following Takeda further we let $W$ be the closure of $B^{*}$ in the weak operator topology. He shows how $W$ can be identified with $B^{* *}$ so that if $F \in B^{* *}$ corresponds to the operator $T$ and $f$ is given by (7.1) then

$$
F(f)=\sum_{k=1}^{n}\left(T\left(\xi_{k}\right), \eta_{k}\right) .
$$

A consequence is that the $w^{*}$-topology on $B^{* *}$ is the same as the weak operator topology on $W$. Now consider the multiplication in $B^{* *}$ induced by the operator multiplication $T_{1} T_{2}$ of two operators in $W$ continuous in each factor in the weak operator topology if the other factor is held fixed. It follows by a remark of Arens [2, p. 844] that the multiplication in $B^{* *}$ corresponding to the operator multiplication in $W$ must be the Arens multiplication in $B^{* *}$. Furthermore the multiplication in $B$ is regular [2, Theorem 3.3].

7.2. Corollary. Let $B$ be a complex commutative semi-simple Banach algebra which is complete in its spectral radius norm. Then 
$B^{* *}$ is commutative and semi-simple.

Proof. As an example consider $A$ the Banach algebra of all complexvalued functions continuous in $|z| \leqq 1$ and analytic in $|z|<1$ where $\|f\|=\sup |f(z)|$. Corollary 7.2 asserts without further computation that $A^{* *}$ is commutative and semi-simple.

From the hypotheses on $B$ we may assume that $B$ is a closed subalgebra of the commutative $B^{*}$-algebra $C(\mathfrak{M})$ of all complex continuous functions vanishing at infinity on the space $\mathfrak{M}$ of regular maximal ideals of $B$. As noted in Theorem 7.1 the multiplication in $C(\mathfrak{M})$ is regular, so that, $C(\mathfrak{M})^{* *}$ is both commutative and semi-simple. By Corollary 6.3, $B^{* *}$ is algebraically isomorphic to a subalgebra of $C(\mathfrak{M})^{* *}$.

\section{REFERENCES}

1. Richard Arens, Operations induced in function classes, Monat. für Math., 55 (1951), $1-19$.

2. The adjoint of a bilinear operation, Proc. Amer. Math. Soc., 2 (1951), 839848.

3. M. M. Day, Amenable semigroups, Ill. J. of Math., 1 (1957), 509-544.

4. J. Dixmier, Sur un théorème de Banach, Duke Math. J., 15 (1948), 1057-1071.

5. N. Dunford and J. Schwartz, Linear Operators, Part I. Interscience Publishers, Inc., New York, 1958.

6. I. M. Gelfand, D. A. Raikov and G. E. Šilov, Commutative normed rings, Amer. Math. Soc., Translations Series 25 (1957), 115-220.

7. L. H. Loomis, An introduction to harmonic analysis, D. Van Nostrand Co., New York, 1953.

8. W. Maak, Fastperiodische Functionen, Springer-Verlag, Berlin, 1950.

9. M. A. Naimark, Normed rings, Moscow (1956). Russian.

10. Walter Rudin, Measure algebras on abelian groups, Bull. Amer. Math. Soc., 65 (1959), 227-247.

11. V. Šmulian, On multiplicative functionals in certain special normed rings, C. R. Acad. Sci. URSS (Doklady) 26 (1940), 13-16.

12. Z. Takeda, Conjugate spaces of operator algebras, Proc. Jap. Acad., 30 (1954), 90-95.

13. André Weil, L'intégation dans les groups topologiques et ses applications, Hermann et Cie, Paris, 1953.

UNIVERSITY OF OREGON 


\title{
PACIFIC JOURNAL OF MATHEMATICS
}

\section{EDITORS}

\author{
Ralph S. Phillips \\ Stanford University \\ Stanford, California \\ F. H. BrownelL \\ University of Washington \\ Seattle 5 , Washington
}

A. L. Whiteman

University of Southern California

Los Angeles 7. California

L. J. PAIGE

University of California

Los Angeles 24, California

\section{ASSOCIATE EDITORS}

\author{
E. F. BECKENBACH \\ T. M. CHERRY
} \\ D. DERRY \\ M. OHTSUKA \\ UNIVERSITY OF BRITISH COLUMBIA \\ CALIFORNIA INSTITUTE OF TECHNOLOGY \\ UNIVERSITY OF CALIFORNIA \\ MONTANA STATE UNIVERSITY \\ UNIVERSITY OF NEVADA \\ NEW MEXICO STATE UNIVERSITY \\ OREGON STATE COLLEGE \\ UNIVERSITY OF OREGON \\ OSAKA UNIVERSITY \\ UNIVERSITY OF SOUTHERN CALIFORNIA
}

H. L. ROYDEN

E. SPANIER
E. G. STRAUS

F. WOLF

\section{SUPPORTING INSTITUTIONS}

\author{
STANFORD UNIVERSITY \\ UNIVERSITY OF TOKYO \\ UNIVERSITY OF UTAH \\ WASHINGTON STATE COLLEGE \\ UNIVERSITY OF WASHINGTON \\ AMERICAN MATHEMATICAL SOCIETY \\ CALIFORNIA RESEARCH CORPORATION \\ HUGHES AIRCRAFT COMPANY \\ SPACE TECHNOLOGY LABORATORIES \\ NAVAL ORDNANCE TEST STATION
}

Mathematical papers intended for publication in the Pacific Journal of Mathematics should be typewritten (double spaced), and the author should keep a complete copy. Manuscripts may be sent to any one of the four editors. All other communications to the editors should be addressed to the managing editor, L. J. Paige at the University of California, Los Angeles 24, California.

50 reprints per author of each article are furnished free of charge; additional copies may be obtained at cost in multiples of 50 .

The Pacific Journal of Mathematics is published quarterly, in March, June, September, and December. The price per volume (4 numbers) is $\$ 12.00$; single issues, $\$ 3.50$. Back numbers are available. Special price to individual faculty members of supporting institutions and to individual members of the American Mathematical Society: $\$ 4.00$ per volume; single issues, $\$ 1.25$.

Subscriptions, orders for back numbers, and changes of address should be sent to Pacific Journal of Mathematics, 103 Highland Boulevard, Berkeley 8, California.

Printed at Kokusai Bunken Insatsusha (International Academic Printing Co., Ltd.), No. 6 , 2-chome, Fujimi-cho, Chiyoda-ku, Tokyo, Japan.

PUBLISHED BY PACIFIC JOURNAL OF MATHEMATICS, A NON-PROFIT CORPORATION

The Supporting Institutions listed above contribute to the cost of publication of this Journal, but they are not owners or publishers and have no responsibility for its content or policies. 


\section{Pacific Journal of Mathematics}

\section{Vol. 11, No. $3 \quad$ BadMonth, 1961}

Errett Albert Bishop, A generalization of the Stone-Weierstrass theorem ..........

Hugh D. Brunk, Best fit to a random variable by a random variable measurable with

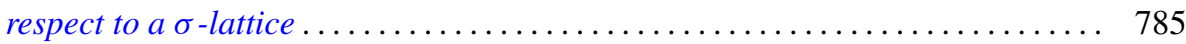

D. S. Carter, Existence of a class of steady plane gravity flows . . . . . . . . . . 803

Frank Sydney Cater, On the theory of spatial invariants ............... 821

S. Chowla, Marguerite Elizabeth Dunton and Donald John Lewis, Linear

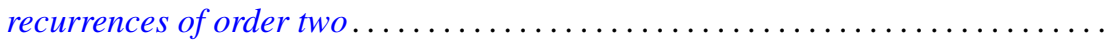

Paul Civin and Bertram Yood, The second conjugate space of a Banach algebra as

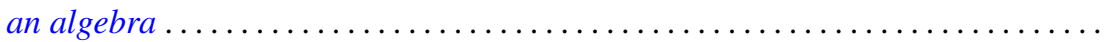

William J. Coles, Wirtinger-type integral inequalities .

Shaul Foguel, Strongly continuous Markov processes ....................

David James Foulis, Conditions for the modularity of an orthomodular lattice ...... Jerzy Górski, The Sochocki-Plemelj formula for the functions of two complex variables.

John Walker Gray, Extensions of sheaves of associative algebras by non-trivial

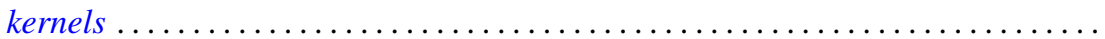

Maurice Hanan, Oscillation criteria for third-order linear differential equations .... 919 Haim Hanani and Marian Reichaw-Reichbach, Some characterizations of a class of unavoidable compact sets in the game of Banach and Mazur .............

John Grover Harvey, III, Complete holomorphs . . . . . . . . . . . . . . . . 961 Joseph Hersch, Physical interpretation and strengthing of M. Protter's method for vibrating nonhomogeneous membranes; its analogue for Schrödinger's

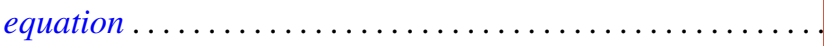

James Grady Horne, Jr., Real commutative semigroups on the plane...

Nai-Chao Hsu, The group of automorphisms of the holomorph of a group...

F. Burton Jones, The cyclic connectivity of plane continua

John Arnold Kalman, Continuity and convexity of projections and barycentric

coordinates in convex polyhedra

Samuel Karlin, Frank Proschan and Richard Eugene Barlow, Moment inequalities of

Pólya frequency functions .

Tilla Weinstein, Imbedding compact Riemann surfaces in 3-space. .

Azriel Lévy and Robert Lawson Vaught, Principles of partial reflection in the set

theories of Zermelo and Ackermann

Donald John Lewis, Two classes of Diophantine equations .....

Daniel C. Lewis, Reversible transformations . . .

Gerald Otis Losey and Hans Schneider, Group membership in rings and

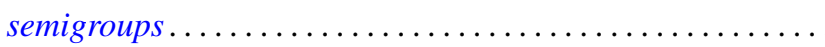

M. N. Mikhail and M. Nassif, On the difference and sum of basic sets of

polynomials.

Alex I. Rosenberg and Daniel Zelinsky, Automorphisms of separable algebras .

Robert Steinberg, Automorphisms of classical Lie algebras .... 